\title{
Simultaneous and Direct Identification of Thermophysical Properties for Orthotropic Materials
}

\author{
Elissa EL RASSY ${ }^{1}$, Yann BILLAUD ${ }^{1, *}$, Didier SAURY ${ }^{1}$ \\ ${ }^{1}$ Institut Pprime UPR CNRS 3346 - CNRS / ENSMA / Université de Poitiers, 1 avenue Clément Ader, B.P. \\ 40109, F86961, Futuroscope Chasseneuil CEDEX, France \\ *(Corresponding author: yann.billaud@ensma.fr)
}

\begin{abstract}
A direct and simultaneous estimation method of the main three dimensional thermal diffusivity tensor $\left(a_{x}, a_{y}, a_{z}\right)$ of orthotropic opaque materials, is presented in this paper. This method consists in coupling a non-intrusive and unique 3D flash experiment with a transient nonlinear inverse heat transfer technique. A short and non-uniform excitation is applied on the surface of an orthotropic material using a $\mathrm{CO}_{2}$ laser, while the front face temperature cartography is measured over time by an IR camera. The inverse problem developed in the present study is based on the minimization of the least-squares criterion between the outputs of a 3D thermal quadrupoles model, and the experimental measurements. In order to properly estimate the thermal diffusivities, parameters related to the thermal excitation, in terms of shape and intensity, should be also estimated. In addition to that increase in the number of unknown parameters, the discontinuity nature of the excitation justify the choice of an analytical model. Considering the large number of parameters to estimate, as well as the non-linear nature of the problem, a hybrid optimization algorithm combining a stochastic method and deterministic one, is applied. The identification method proposed in this work, named as DSEH (Direct and Simultaneous Estimation using Harmonics), is validated using an isotropic opaque material of known properties. Finally, the method is used on an orthotropic carbon fiber composite, commonly used in industries, thanks to its thermal and mechanical characteristics. The results are compared to other methods and shown to be in a good agreement with the literature values. The parameters identification is then completed by a sensitivity analysis, and evaluated in terms of robustness, accuracy, and time consumption.
\end{abstract}

KEYWORDS: Thermophysical properties, thermal quadrupoles, integral transforms, orthotropic materials, flash method, inverse problems, parameters estimation, IR thermography. 


\section{Nomenclature}

\section{Latin Symbols}

$\begin{array}{clc}a_{x}, a_{y}, a_{z} & \text { Thermal diffusivities } & \mathrm{m}^{2} \cdot \mathrm{s}^{-1} \\ C & \text { Thermal capacity } & \mathrm{J} \cdot \mathrm{kg}^{-1} \cdot \mathrm{K}^{-1} \\ F_{x, y} & \text { shape function associated with the laser beam } & \mathrm{m}^{-2} \\ F_{m, n} & \text { Fourier coefficients of the shape function } & \\ h & \text { Overall heat transfer coefficient } & \mathrm{W} \cdot \mathrm{m}^{-2} \cdot \mathrm{K}^{-1} \\ l_{x}, l_{y}, l_{z} & \text { Sample's dimensions } & \mathrm{m} \\ M, N & \text { Harmonics maximum indices } & \mathrm{J} \\ p & \text { Laplace variable } & \mathrm{J} \\ Q & \text { amount of energy absorbed by the sample } & \\ R_{m, n} & \text { Excitation factor } & \mathrm{m} \\ S & \text { Reduced Sensitivity } & \mathrm{K} \\ r & \text { Laser spot radius } & \mathrm{s} \\ T & \text { Temperature elevation } & \\ u(t) & \text { Time shape function of the laser beam } & \\ u(p) & \text { Laplace transform of the time shape function } & \\ X_{m}(x) & \text { Basis function in the x-plane } & \end{array}$

\section{Greek symbols}

$\begin{array}{clc}\alpha_{m}, \beta_{n} & \text { harmonics pulsation } & \mathrm{rad} . \mathrm{m}^{-1} \\ \rho & \text { Density } & \mathrm{kg} \mathrm{m} \mathrm{m}^{-3} \\ \lambda_{x}, \lambda_{x}, \lambda_{x} & \text { Thermal conductivities } & \mathrm{W} \cdot \mathrm{m}^{-1} \cdot \mathrm{K}^{-1} \\ \xi_{\mathrm{m}, \mathrm{n}}(t) & \text { Normalized harmonics in time domain } & \mathrm{K} \\ \xi_{\mathrm{m}, \mathrm{n}}(p) & \text { Normalized harmonics in Laplace domain } & \mathrm{K} . \mathrm{s} \\ \theta_{\mathrm{m}, \mathrm{n}}(p) & \text { Harmonics in Laplace domain } & \mathrm{K} . \mathrm{m}^{2} . \mathrm{s} \\ \phi_{f} & \text { Overall heat losses on front face } & \mathrm{J} \\ \phi_{b} & \text { Overall heat losses on back face } & \mathrm{J} \\ \phi_{m, n}^{e x}(p) & \text { Excitation in the Fourier and Laplace domains } & \mathrm{J}\end{array}$




\begin{tabular}{|c|c|c|}
\hline$\phi_{x, y}^{e x}(t)$ & Excitation in the physical and time domains & $W \cdot m^{-2}$ \\
\hline B & Parameters vector to be estimated & \\
\hline$\hat{B}$ & Optimal parameters vector & \\
\hline$\Psi$ & Cost function & \\
\hline
\end{tabular}

\author{
Subscripts \\ $x, y, z \quad$ Cartesian coordinates \\ $m, n \quad$ Spatial Fourier modes \\ Estimators \\ DSEH Direct \& Simultaneous Estimation using harmonics \\ ERH Estimation using Ratio of Harmonics \\ ENH Estimation using Normalization of harmonics \\ MSEH Multiple Steps Estimation using Harmonics
}

\title{
1. Introduction
}

The continuous emergence of new materials with complex structures (anisotropic, multilayers, porous, and heterogeneous) in various industrial sectors, notably in aeronautics, appeals their thermal characterization to ensure the control and the modelling of the heat transfers through the processes. As a part of the thermal characterization, the identification of thermophysical properties of such materials has taken, from many years, a significant and increasing concern. There are several methods dedicated to the estimation of thermophysical properties. These methods may be classified according to many criteria, as presented by Degiovanni [1] and Rodiet [2] who realized a review of main methods : i) the estimation regime (steady, transient, periodic); ii) the estimated parameters (diffusivity, effusivity, conductivity, heat capacity); iii) the excitation method (photothermal, electrothermic) and characteristics which can be spatial (parabolic, Gaussian, random, rectangular...) and temporal (pulse, step, periodic); iv) the measurement technique (with or without contact) and spatial extent (local or extended); v) the measured quantities: temperatures or heat flux,; vi) the geometry of the problem (1D, 2D, 3D), etc.

Among these methods, the flash method, proposed by Parker [3], is now one of the standard techniques for measuring solid thermal properties. This transient approach was originally based on a homogeneous short 
duration light pulse with a local measurement of the temperature in the back face of the sample. The indepth thermal diffusivity is determined by means of a one dimensional conductive heat transfer model. This technique has been widely improved over the past 50 years. Improvements will be discussed, here, according to the geometry of the problem.

The extension of this 1D approach into 2D, was first motivated to take into account non uniform excitation [4], then it was improved by Amazouz [5] and applied by Degiovanni [6] in order to estimate in-plane diffusivity of anisotropic solid materials. This technique has been also applied to the estimation of both inplane and in-depth diffusivities of anisotropic materials, or specifically orthotropic materials [7], i.e. having principle diffusivities directions aligned with the Cartesian coordinate system. The flash method is then used in cylindrical coordinates to separately estimate radial and axial diffusivities [8], [9].

Furthermore, the extension into 3D identification methods leads to some achievements, notably the compatibility between the model and the experimental conditions, which is usually a difficult task. The pioneer works conducted by Sawaf [10] are an illustration of this constraint. The attempt to estimate the each of three main diffusivities of a sample by subjecting, constant heat flux on one face, while keeping other faces insulated, is unattainable experimentally. The compromise between the experimental accuracy and the capability of the model has to be consistent. The consideration of new boundary conditions, with the aim of a best the environmental conditions control of the experiments, combined with the increase in the calculation capacity, brings new estimation methods based on numerical simulations. Several methods that solve the 3D heat conduction problem using the finite difference or finite volume techniques [11],[12] the finite element method [13]-[15] the boundary element method [16],[17] and the singular boundary method [18], have proven to be highly time consuming.

In this context, the development of estimation methods, whether in 2 or 3-D coordinates, based on the analytical resolution of heat transfer problem is still relevant. Methods, such as the Estimation using Ratio of harmonics (ERH) proposed by Philippi [19] then developed by Remy [20],[21], in addition to the Estimation using Normalization of harmonics (ENH) [22]-[24] has been developed and successfully used for the identification of the diffusivities in the plane $\left(a_{x}\right.$ and $\left.a_{y}\right)$.

Another method, based on the resolution of the conductive heat transfer by means of Fourier transform, is known as MSEH [25] or "Multiple Steps Estimation using harmonics". This technique conducts a step by step estimation starting by the estimation of the in-depth diffusivity from the average field, which is the most sensitive parameter to environmental noise. The estimated in-depth diffusivity is then used to identify the transverse diffusivities $\left(a_{x}\right.$ and $\left.a_{y}\right)$. Although giving relevant results, this method may experience a low 
accuracy due to its sequential nature. The multi-step identification strategy is also used by Rodiet [26] to estimate the orthotropic material diffusivity tensor using the average temperatures in the in-plane directions.

The above discussion has been conducted from the direct model point of view and its exploitation via the identification procedure. For clarity reasons, the experimental point of view has been evaded. One should notice that some works are purely theoretical, others are based on experiments that rely on intrusive measurements (e.g. thermocouples) [8], and/or intrusive heating source [11]-[13],[27]. Also, the experimental protocol may be sophisticated, where some authors have developed methods based on two experiments (e.g. consisting in sample rotation) or on the duplication of the set of device (e.g. two series of laser and IR camera)[14],[15].

The main feature of this work relies on the combination of the direct and simultaneous estimation of the 3 principle components of the diffusivity tensor using an analytical 3D transient model and a unique and nonintrusive experiment, in terms of both excitation and measurements. The direct model, relying on the Fourier transform that allows the exploitation of a large amount of spatial data, is the key element of the estimation method referred in this paper as "Direct and Simultaneous Estimation using Harmonics" (DSEH). The importance of direct identification method was firstly mentioned by Ruffio in a comparative study [28].

Considering the complexity, the non-linearity of the inverse problem, as well as the large number of parameters to estimate, gradient based methods that would find local optimums cannot be applied directly in this case. A global search algorithm is then required and the stochastic methods appear to be a good choice for this purpose. Several existing heuristic and evolutionary algorithms are successfully applied in many engineering fields [29]-[37] to find a global optimum, and can be good candidates in this study. In order to provide a best possible estimation, a hybrid optimization algorithm combining a stochastic method and deterministic one (gradient based), is applied here.

The present article focus on the development of an analytical model, based on the thermal quadrupole approach [38] which is conducted to predict temperature evolution at the front faces of orthotropic materials that are exposed to a Dirac type imposed flux and subject to natural cooling. The corresponding experimental setup, composed of a $\mathrm{CO}_{2}$ laser used to generate the localized thermal excitation and an IR camera used to record the temperature evolution on the exposed face, is detailed and followed by a description of the experimental protocol. The choice of the current optimization algorithm, as well as the objective function are discussed and evaluated using synthetic noisy data. The identification method is then applied on real samples with isotropic and orthotropic properties. Moreover, the identification results are compared with results from well-established methods as ENH and MSEH, previously discussed. Finally, accuracy, 
robustness and time consumption of the method are investigated by means of a sensitivity and improvement study.

\section{Physical configuration and mathematical model}

The main objective being the determination of the 3 thermal diffusivity components of orthotropic materials, the configuration to handle has to be tridimensional. The procedure consists in exposing samples of such material to an impulse type excitation, produced by a $\mathrm{CO}_{2}$ laser, and localized on the front face $(\mathrm{z}=0)$. This non uniform thermal excitation generates a moderate local temperature elevation that diffuses in the sample, in such a way to keep the thermophysical properties constant and independent on the temperature. The resultant temporal and spatial evolution of the temperature through the opaque and homogenous material is described by a partial differential equation, completed by equations describing the initial and boundaries conditions. The front and rear faces are exposed to convection and radiation losses, described by a linearized and global heat exchange coefficient $h$. Nonetheless, the lateral faces are assumed to be thermally isolated, and this condition will be discussed later on. The set of differential partial equations (1.a-2.f) describing the system is given hereafter as well as in Figure 1.

$$
\left\{\begin{array}{ccc}
\frac{\partial T}{\partial t}=a_{x} \frac{\partial^{2} T}{\partial x^{2}}+a_{y} \frac{\partial^{2} T}{\partial y^{2}}+a_{z} \frac{\partial^{2} T}{\partial z^{2}} & \text { at } & z \in[0, l z], t>0 \\
-\lambda_{x} \frac{\partial T}{\partial x}=0 & \text { at } & x=0 \text { and } x=l_{x}, t>0 \\
-\lambda_{y} \frac{\partial T}{\partial y}=0 & \text { at } & y=0 \text { and } y=l_{y}, t>0 \\
-\lambda_{z} \frac{\partial T}{\partial z}=-h T+\phi(x, y, t) & \text { at } & z=0, t>0 \\
-\lambda_{z} \frac{\partial T}{\partial z}=h T & \text { at } & z=l_{z}, t>0 \\
T(x, y, z)=0 & \text { at } & t=0
\end{array}\right.
$$

Please notice that the system of equation (3.a-4.f) relies on the relative temperature T, i.e. the difference between the local temperature and the initial temperature $\mathrm{T}(\mathrm{t}=0)$. Before each experiment, the sample is supposed to be at thermal equilibrium with the environment, i.e. the initial temperature is considered to be equal to the temperature of the surrounding environment. 


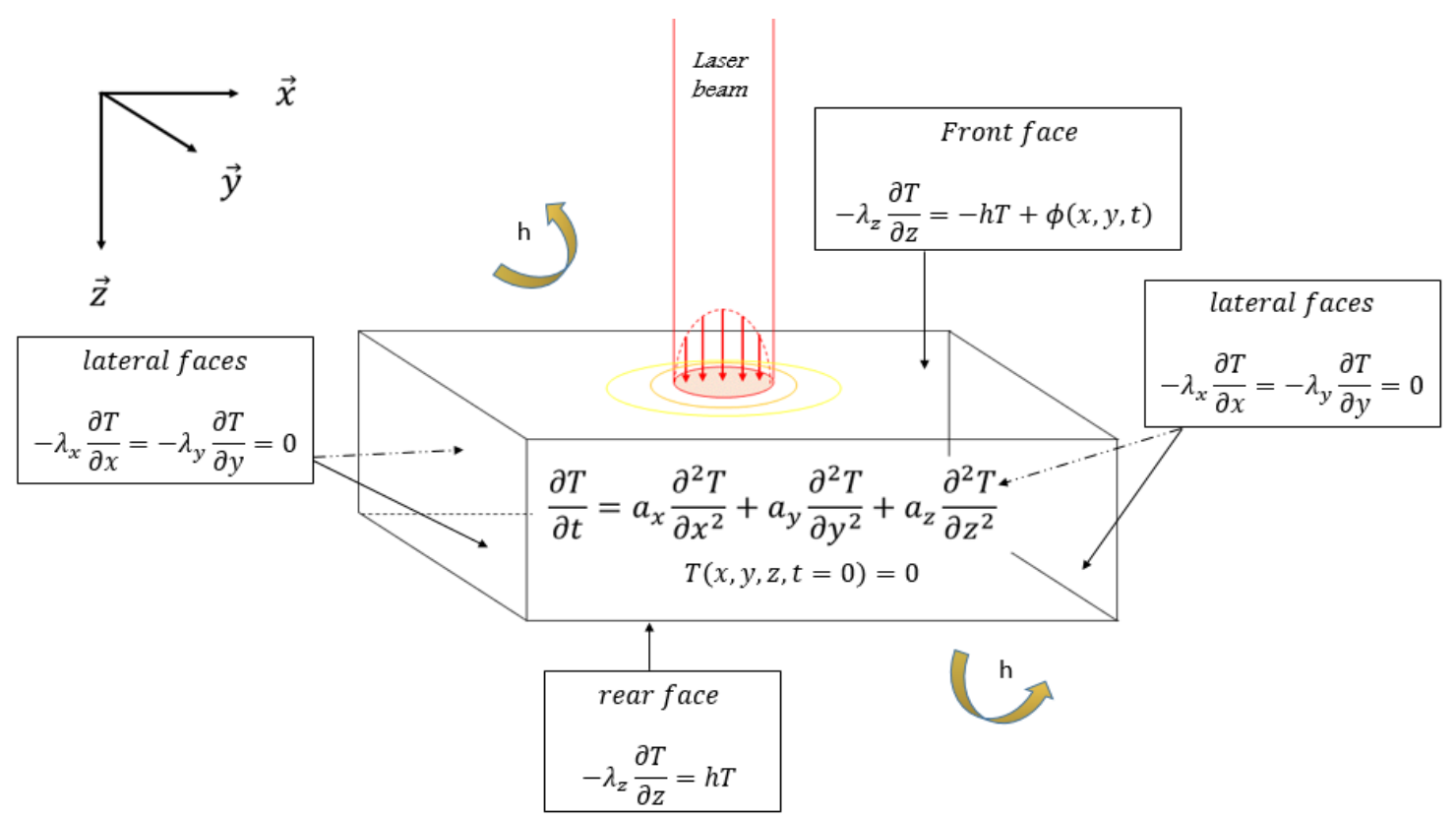

Figure 1 : Physical configuration, mathematical modelling and boundary conditions in the real domain.

The parameters identification relies on the post-treatment of the temperature evolution at the sample front face. This treatment consists in an integral transformation, applied to both experimental measurements and direct model outputs, in Fourier cosine spaces.

Fourier transformations result in the appearance of harmonics, $\theta_{m, n}$, whose main advantages are a fast treatment of the direct simulation and a capacity of noise filtering by selection of the relevant harmonics.

Given that the harmonics of low spatial frequencies hold the largest quantity of information related to diffusivities, the exploited harmonics in this study will be the first $M \times N \leq 6$, even modes.

These harmonics (Fourier-Laplace domain) are defined as:

$$
\theta_{m, n}(z, p)=\int_{0}^{\infty} \int_{0}^{l_{y}} \int_{0}^{l_{x}} T(x, y, z, t) \cdot X_{m}(x) \cdot Y_{n}(y) \cdot e^{-p t} \cdot d x \cdot d y \cdot d t
$$

Where $X_{m}(x)=\cos \left(m \cdot \pi \cdot \frac{x}{l_{x}}\right), Y_{n}(y)=\cos \left(n \cdot \pi \cdot \frac{y}{l_{y}}\right)$ are the basis function defining the FourierCosine space. Those functions have been chosen according to the boundary conditions considered in this study. 
It is worth mentioning that the odd harmonics (i.e. where $\mathrm{m}$ or $\mathrm{n}$ are odd), are not taken into account, since they are quasi-null (three order of magnitude lower than the even harmonics values), due to the symmetric characteristic of the excitation. Thus, the information is concentrated only on even harmonics which are then considered for the identification procedure. Following the formalism of the thermal quadrupoles method applied on the system, the relative front-face temperature evolution, is given by solving the system of equations shown in Figure 2.
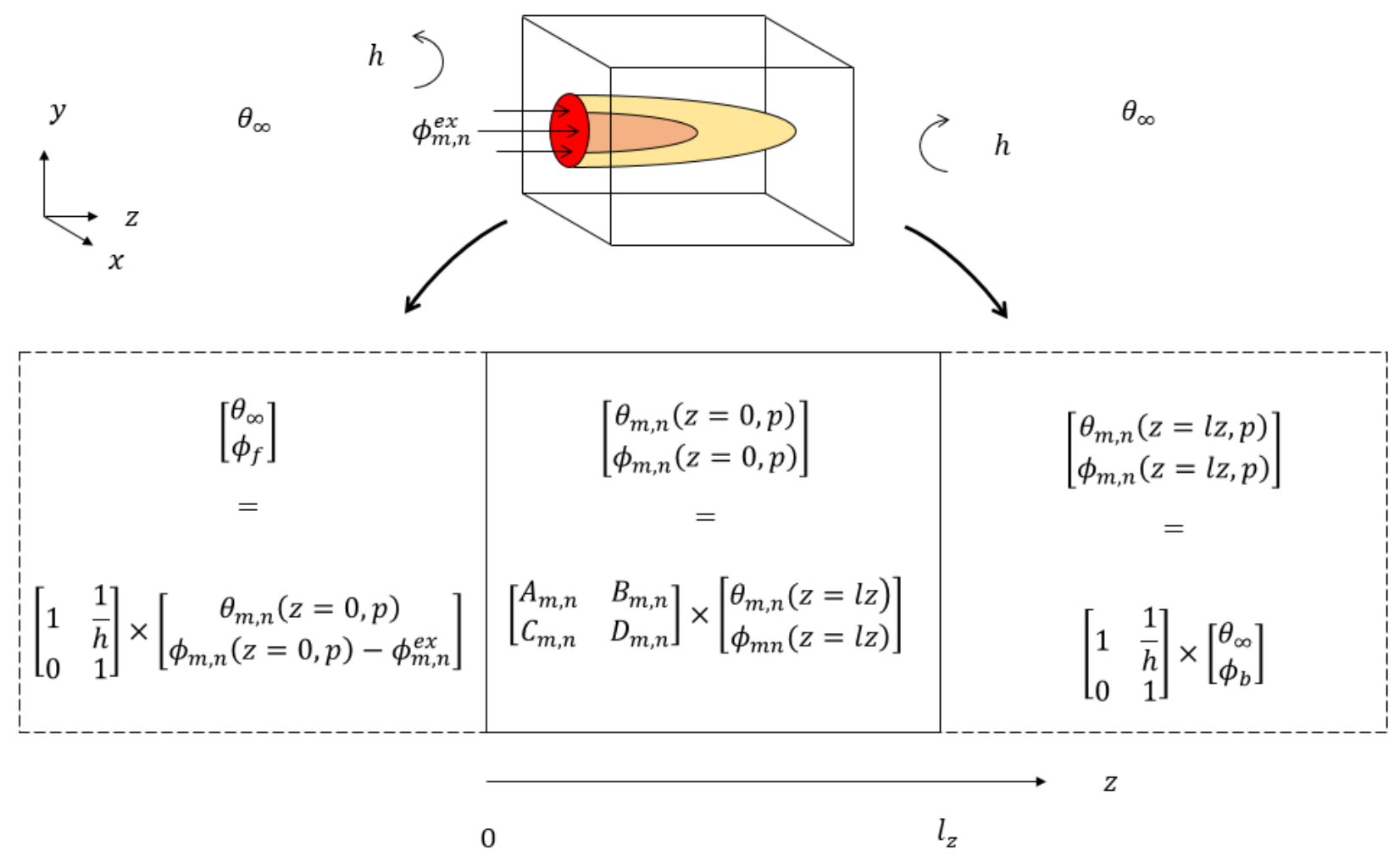

Figure 2 : Physical configuration, mathematical modelling and boundary conditions in the xy-Fourier and t-Laplace domain.

The system of equations in Figure 2 lead to:

$$
\left[\begin{array}{c}
\theta_{m, n}(z=0, p) \\
\emptyset_{m n}^{e x}-h \cdot \theta_{m, n}(z=0, p)
\end{array}\right]=\left[\begin{array}{ll}
A_{m, n} & B_{m, n} \\
C_{m, n} & D_{m, n}
\end{array}\right] \times\left[\begin{array}{c}
\theta_{m, n}(z=l z, p) \\
h \cdot \theta_{m, n}(z=l z, p)
\end{array}\right]
$$

Given that the temperatures of the environment in the Fourier-Laplace domain, are equal on both sides of the sample, with $\theta_{\infty}=0$. The conductive heat behavior is piloted by the quadrupole terms defined as [38] : 


$$
\left[\begin{array}{ll}
A_{m, n} & B_{m, n} \\
C_{m, n} & D_{m, n}
\end{array}\right]=\left[\begin{array}{cc}
\cosh \left(l_{z} \cdot K_{m n}\right) & \sinh \left(l_{z} \cdot K_{m n}\right) /\left(\rho C \cdot a_{z} \cdot K_{m n}\right) \\
\rho C \cdot a_{z} \cdot K_{m n} \cdot \sinh \left(l_{z} \cdot K_{m n}\right) & \cosh \left(l_{z} \cdot K_{m n}\right)
\end{array}\right]
$$

where $K_{m n}=\sqrt{\frac{p}{a_{z}}+\alpha_{m}^{2} \frac{a_{x}}{a_{z}}+\beta_{n}^{2} \frac{a_{y}}{a_{z}}}, \alpha_{m}=\frac{m \cdot \pi}{l_{x}}$ and $\beta_{n}=\frac{n \cdot \pi}{l_{y}}$.

$\phi^{e x}$ designates the excitation, that can be decomposed into a product of its magnitude(intensity) and two functions, one representing the space shape $F_{x, y}$ and the other characterizes the time form $u(t)$. Thus, $\phi_{x, y, t}^{e x}=Q \cdot F_{x, y} \cdot u(t)$.

Projected into the Laplace and Fourier domains, it gives: $\emptyset_{m n, p}^{e x}=Q \cdot F_{m, n} \cdot u(p)$ with $F_{m, n}=$ $\int_{0}^{l_{y}} \int_{0}^{l_{x}} F_{x, y} \cdot X_{m}(x) \cdot Y_{n}(y) \cdot d x \cdot d y$, and $u(p)=1$ for an impulse of Dirac type. The dimensionless parameters $F_{m, n}$ and $u(p)$ represents the shape and the time factors, respectively.

Following equation (6), normalized by $\left(l_{x} \cdot l_{y}\right)$, the front face harmonics in the Laplace domain yields to:

$$
\xi_{m, n}(p)=\frac{\theta_{m, n}(p)}{l_{x} \cdot l_{y}}=\frac{\frac{Q \cdot F_{m, n} \cdot u(p)}{l_{x} \cdot l_{y}}\left(\cosh \left(e \cdot K_{m n}\right)+\frac{\sinh \left(e \cdot K_{m n}\right)}{\lambda_{z} \cdot K_{m n}} h\right)}{\lambda_{z} \cdot K_{m n} \cdot \sinh \left(e \cdot K_{m n}\right)+2 \cdot \cosh \left(e \cdot K_{m n}\right) h+\frac{\sinh \left(e \cdot K_{m n}\right)}{\lambda_{z} \cdot K_{m n}} h^{2}}
$$

Calculation time obtained for one harmonic for a $\mathrm{p}$ vector size of 1200 (i.e. about $24 \mathrm{~s}$ of physical time in the present work) is inferior to $10^{-3} \mathrm{~s}$. The approximative number of harmonics used for identification is 16 , which make the typical direct calculation time used for the identification is about $t_{C P U} \approx 10^{-2} \mathrm{~s}$, which is compatible with a stochastic identification approach.

\section{Inverse problem and estimation strategy}

In this part, the global inverse strategy and the estimation procedure that lead to the parameters identification, are discussed and detailed. It involves three main elements constituting the concept of an inverse problem: i) the vector of parameters to identify, ii) the cost function and iii) the optimization algorithm. The buildup method is validated against synthetic data.

\subsection{Parameters vector}

The inverse problem treated here is rather complex because, besides the estimation of the thermal diffusivities tensor, the parameters related to the excitation has to be estimated due to their influence on the system response. The parameters vector to identify is then $B=\left[a_{x}, a_{y}, a_{z}, R_{0,0}, R_{0,2}, R_{2,2}, \ldots R_{m, n} \ldots, R_{M, N}\right]$ with $R_{m, n}=Q \cdot F_{m, n}$ corresponding to the heat flux distribution in the Fourier modes space. In this way, the size of parameters vector that should be estimated depends on the number of modes chosen to describe 
the excitation. The number of even modes depends on both, the shape of the laser beam and the minimum number of modes required to ensure a proper direct simulation. For symmetrical reason, $\mathrm{M}$ and $\mathrm{N}$ should be chosen equal. The number of parameters to be estimated is then equal to $3+\left(\frac{M}{2}+1\right)^{2}$ when considering only even modes.

\subsection{Cost function}

The cost function, also known as objective function, is defined as the quadratic deviation between the measured signal and the signal predicted by the direct physical model. Noting that the above discussed model is producing output signal as harmonics in the Laplace and Fourier-Cosine spaces, it is theoretically possible to compare signals in 4 probable domains:
(i) the physical domain $T(x, y, z, t)$,
(ii) the Laplace domain $T(x, y, z, p)$,
(iii) the Fourier Cosine domain $\xi_{m, n}(t)$,
(iv) both the Fourier-Cosine and Laplace domains $\xi_{m, n}(p)$.

Extensive trials previously conducted demonstrate that the minimization in the time harmonics space, i.e. domain (iii), is more suitable, both in terms of accuracy (measurement noise filtering) and identification speed (spatial image compression). Concerning the latter and as already discussed, the direct analytical model is quasi instantaneous $\left(t_{C P U} \sim 10^{-2} s\right)$, which is a benefit compared to numerical models used in some works presented in the literature. Thus, the estimator dedicated for the minimization of the cost function, could be written as follows:

$$
\psi=\min _{\beta} \sqrt{\sum_{m=0}^{M} \sum_{n=0}^{N}\left[\xi_{m, n}(b, t)-\xi_{m, n}^{e x p}(t)\right]^{2}}
$$

In this method, the considered harmonics are equally weighted. The first term of equation $(9), \xi_{m, n}(8, t)$, corresponds to the temporal normalized harmonics, achieved by the Laplace inversion which is applied to the direct model output in equation $(8), \xi_{m, n}(p)$. This inversion is successfully realized using De Hoog algorithm [39], that was found to be the most appropriate in the current problem. The second term, $\xi_{m, n}^{\exp }(t)$ represents the observables issued from temporal measurement of the front face temperatures, and projected in Fourier Cosine space. 


\subsection{Optimization algorithm}

Identification of the optimum parameters vector $\widehat{B}$, that minimizes the objective function $\sum_{m=0}^{M} \sum_{n=0}^{N}\left[\xi_{m, n}(B, t)-\xi_{m, n}^{\exp }(t)\right]^{2}$, is achieved by a hybrid optimization procedure combining both a stochastic and deterministic method. Among the existing stochastic methods, the Particle Swarm Optimization (PSO) algorithm is chosen for its relative ease of understanding and implementation as well as its relatively low number of parameters to tune. Adding to that the efficiency of this algorithm has been proved in many different engineering fields [40]-[47], and especially when applied for heat transfer problems concept [29]-[31], [48]-[51]. This type of nature inspired and zero order algorithm is well adapted to such problem as each candidate, representing a potential solution of the minimization problem, evolves in a collaborative way inside the domain [52]-[54]. In this approach, a potential solution of the minimization problem is represented by a particle position $P_{i}$. This position will iteratively evolve according to criteria, expressed by the terms on the right hand side of equation (10) . The first term is related to the particle inertia that linearly depends on the previous velocity and an inertial coefficient $w$ that evolves in such a way to promote the exploration in the early stage of the search process. The inertial coefficient is updated at each iteration considering the number of iteration without any changes of the best objective function value. The initial value of $w$ is set to 1.1 , and is modified at each improvement of the best objective function according to the following rules. For any improvement that occurs after 2 or less previous iterations without improvement, the value of $w$ is dividied by a factor 2 . On the contrary, for any improvement that occurs after 5 iterations or more without improvement, the value of $w$ is divided by a factor 2. Moreover, a test is implemented to ensure that the value of $w$ is kept into the range $[0.1-1.1]$. Please note that, higher values of $w$ encourage exploring the search domain, lower values of $w$ speed up convergence. The second term represents the social behavior, in which the particle direction is influenced by the experience (i.e. best $f$ ) shown among the neighborhood of the particle. The third term represents the cognitive behavior, in which the particle direction is influenced by its own experience. These two latter terms are weighted by empirical coefficients $c_{1}$ and $c_{2}$. In order to avoid the algorithm being trapped into a local minima, these terms are completed with coefficients $r_{1}$ and $r_{2}$ whose values, ranging from 0 to 1 , are randomly at each PSO iteration $k$ and for each particle $i$. Once getting the velocity for each component (i.e. parameter to identify, non-indexed for readability reasons), $v_{i}^{k+1}$, the new position of the particle in the search domain, $P_{i}^{k+1}$, is computed.

$$
v_{i}^{k+1}=w \cdot v_{i}^{k}+c_{1} \cdot r_{1}(i, k) \cdot\left[\widehat{N_{l}^{k}}-P_{i}^{k}\right]+c_{2} \cdot r_{2}(i, k) \cdot\left[\widehat{P_{l}^{k}}-P_{i}^{k}\right]
$$




$$
P_{i}^{k+1}=P_{i}^{k}+v_{i}^{k+1}
$$

The parameters driving the behavior of PSO particles and used in the set of equations (10) are commented in Table 1.

\begin{tabular}{|c|c|l|}
\hline PSO parameters & Values & Description \\
\hline $\boldsymbol{w}$ & {$[0.1-1.1]$} & inertia (adaptive) of particles \\
\hline $\boldsymbol{c}_{\mathbf{1}}, \boldsymbol{c}_{\mathbf{2}}$ & 1.49 & acceleration coefficients \\
\hline$\widehat{\boldsymbol{N}_{\boldsymbol{l}}^{\boldsymbol{k}}}$ & - & best experience among the neighbors of the particle $i$ \\
\hline$\widehat{\boldsymbol{P}_{\boldsymbol{l}}^{\boldsymbol{k}}}$ & - & best experience of the particle $i$ \\
\hline $\boldsymbol{r}_{\mathbf{1}}(\boldsymbol{i}, \boldsymbol{k}), \boldsymbol{r}_{\mathbf{2}}(\boldsymbol{i}, \boldsymbol{k})$ & $U(0 ; 1)$ & uniform random vector for each particle $i$ at each iteration $k$ \\
\hline
\end{tabular}

Table 1-Parameters and values used in the PSO algorithm.

This strategy allows an interesting capacity of investigation when the research domain has a high dimension and the model is strongly nonlinear, which is the case in this work.

The result obtained from the PSO step, is then used as initial condition to a gradient based algorithm, namely the interior point method whose mathematical implementation into computer calculation is described in [55]. The method relies on the idea of a barrier function that handled the constraints linked to the search space domain of each unknown parameter. The resulting approximate problem is solved via the well-known Newton's method. When the problem is not locally convex near the current iterate, the problem is solved via a conjugate gradient method. Those deterministic algorithms, involved in the interior point method, are best suited for a local convergence of the solution. Finally, the overall strategy takes advantages of both methods, i.e. the capability of the PSO method to search for the global minimum region and the capability of the interior point method to find the local optimum of the objective function in the global minimum region previously found. Although not shown here for brevity reasons, the feasibility, accuracy and robustness of the minimization method, has been tested using synthetic data generated by the direct model to which a uniform noise ranging from 5 to $10 \%$ of the initial signal was added. In addition, to ensure that the methodology retrieve the initial parameters (the ones used to generate the synthetic data), this preliminary study allows to calibrate the PSO parameters that control the behavior and efficiency of the PSO method (number of particles, inertial weight, cognitive and social parameters ...). One should notice that this strategy, literally known as « inverse crime », allows to validate the optimization method, but in no case the validity of the direct model. 


\section{Experimental setup and results}

In this section, the experimental setup, in terms of procedure and tools, is presented. The proposed identification method is validated on well-known properties isotropic samples of polyamide, and then applied on an orthotropic sample of carbon fibers composite.

\subsection{Experimental procedure}

Figure 3 shows the experimental setup and the equipment used to generate the experimental data. The impulse excitation, produced by a $\mathrm{CO}_{2}$ laser (DIAMOND GEM-Series by Coherent $\mathrm{C}, 10,6 \mu \mathrm{m}$ ), is modulated in power (from $5 \%$ to $100 \%$ of its total capacity of $130 \mathrm{~W}$ ) and time (from $10 \mathrm{~ms}$ to many seconds). The thermal response of the sample is recorded at the front face of the sample during $30 \mathrm{~s}$ by an IR camera (FLIR SC7500) with a $320 \times 256$ pixels resolution at a frequency of $50 \mathrm{~Hz}$.

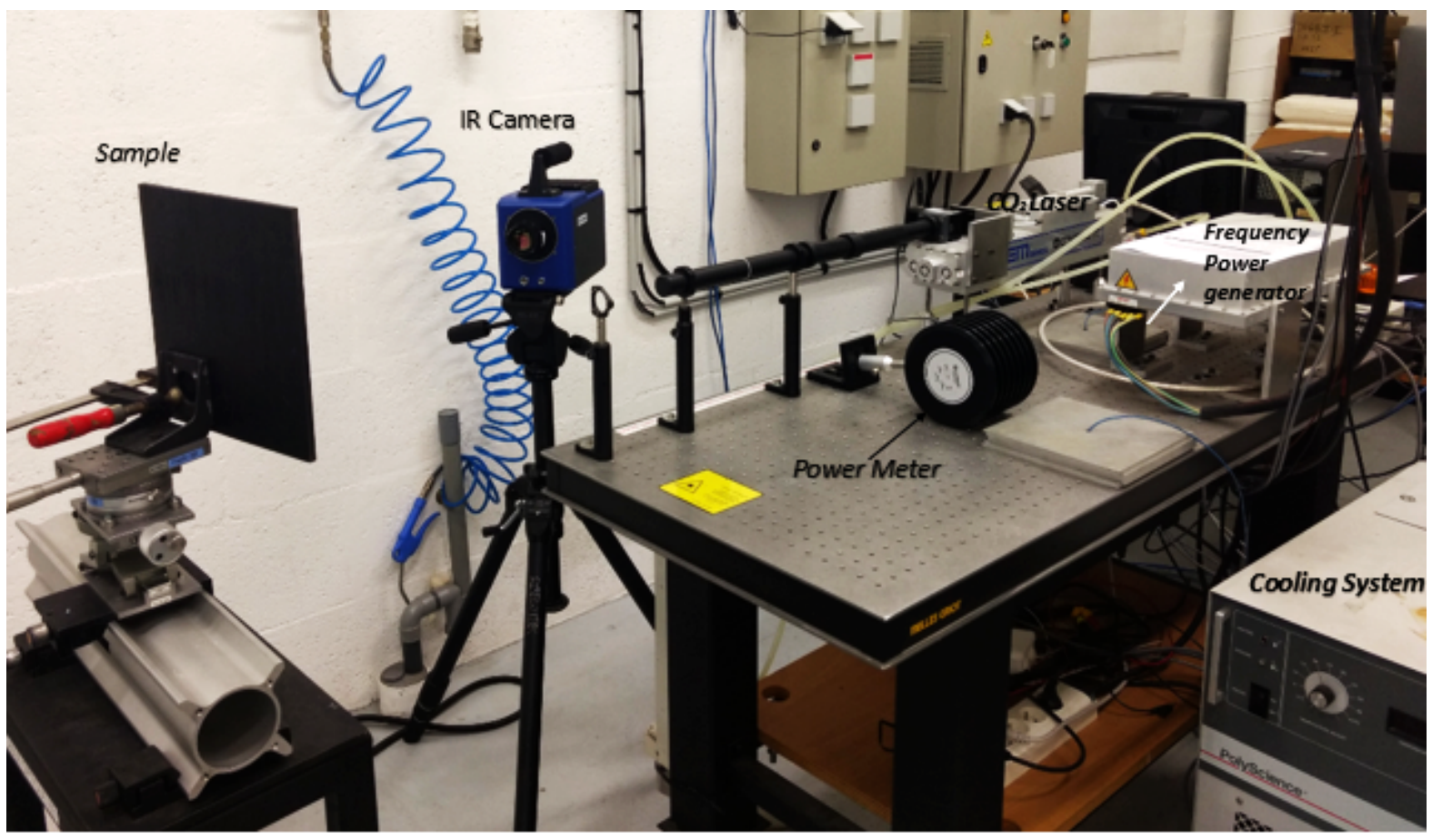

Figure 3: Experimental setup representing the front face flash method.

\subsection{Images processing and exploitation}

The treatment of the raw data consists in,

(i) converting the intensities levels from digital levels (DL) into temperature levels,

(ii) calibrating the data (i.e. definition of the correlation pixel $/ \mathrm{mm}$ and dimension of the exploitation window $l_{x}$ and $l_{y}$ ),

(iii) centering the resultant frames with regard to the laser spot as shown in Figure 4, 
(iv) identifying the excitation moment and subtracting the bottom signal to manipulate with relative temperatures(relative to initial temperature),

(v) projecting the data in Fourier-Cosine space,

(vi) eventually, selecting the data to be exploited.

This procedure leads to the experimental data $\xi_{m, n}^{\text {exp }}(t)$ used in equation (9) for the estimation discussed in the previous section.

As shown on Figure 4, the choice of the exploitation frames, illustrated by the colored frame, is of prime importance. A compromise is required between a sufficient size to respect the boundary conditions of the direct model and a surface area restricted to the zone of interest to avoid the degradation of the data (by dilution of the signal in the background signal).

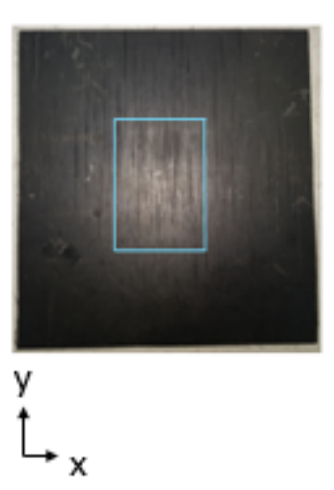

a) Carbon fiber composite sample

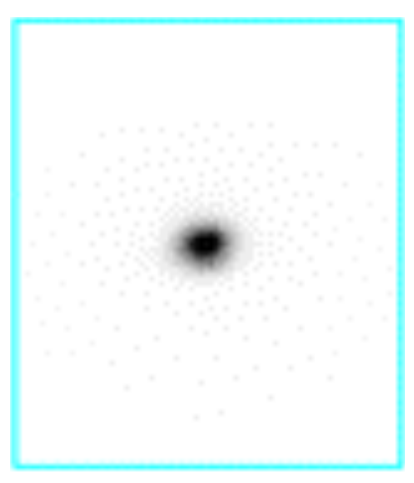

b) $t=t_{0}+0.08 \mathrm{~s}$

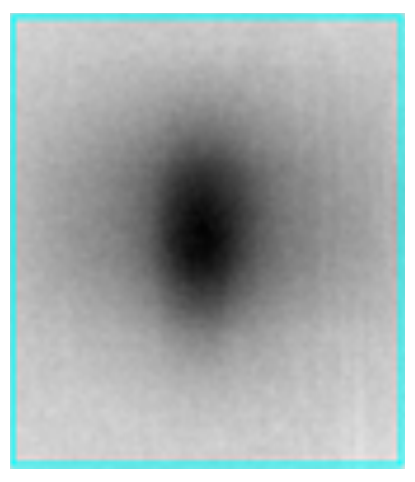

c) $t=t_{0}+8 \mathrm{~s}$

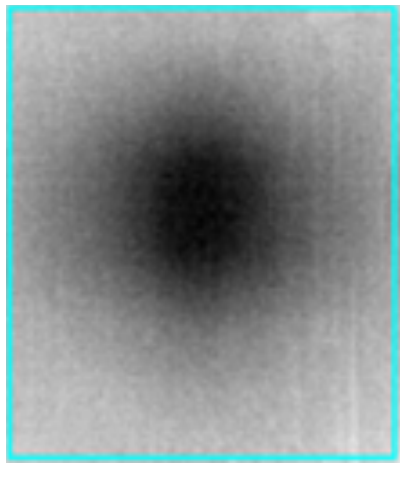

d) $t=t_{\text {end }}$

Figure 4 : Raw pictures cropping $\left(l_{x} \times l_{y}=39.0 \times 45.0 \mathrm{~mm}\right)$ at different time after the laser beam impact at $t_{0}$.

Concerning the boundary conditions, Figure 5 shows the experimental temperature elevation on a composite front surface, $b)$ just a few moments $(0.08 \mathrm{~s})$ after the excitation, c) after a certain time $(8 \mathrm{~s})$ and $\mathrm{d})$ at the time, $t_{\text {end }}$, corresponding to the exploitation limit of experimental data . 
Cite this paper as : E. EL RASSY, Y. BILLAUD, D. SAURY, "Simultaneous and Direct Identification of Thermophysical Properties for Orthotropic Materials", Measurement

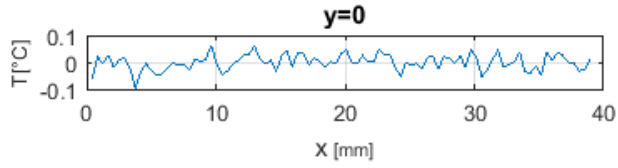

(a)
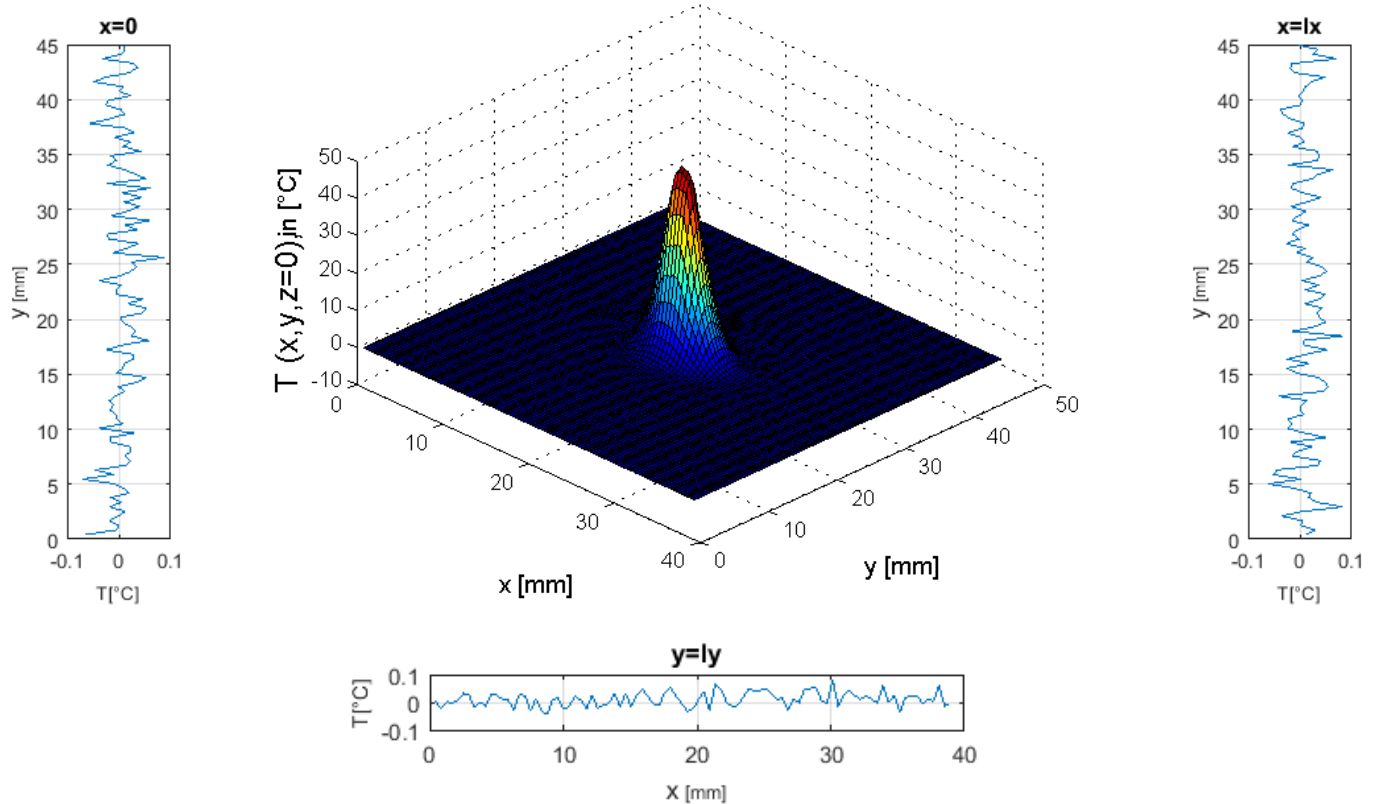

(b)
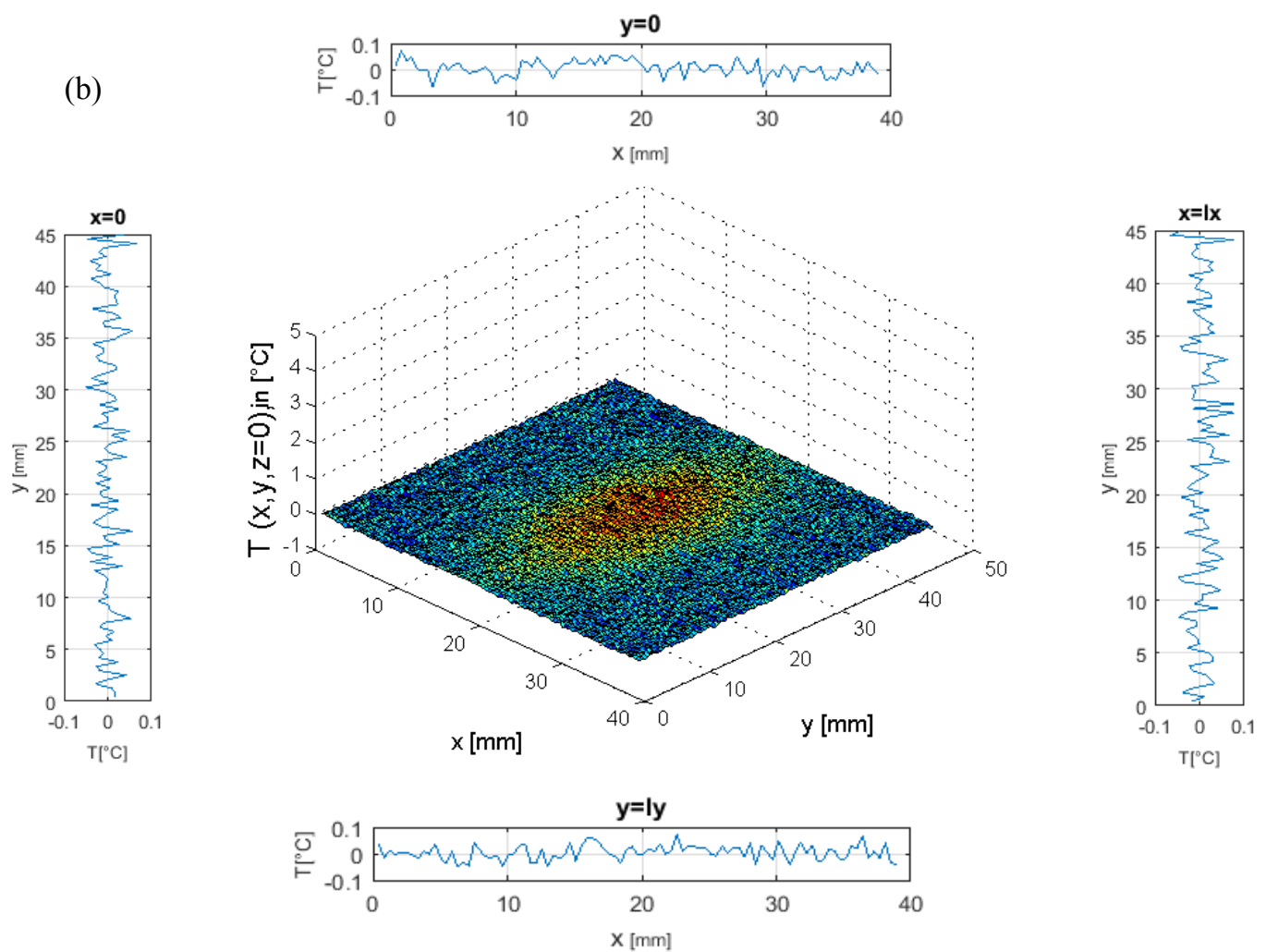

Figure 5 : Experimental temperature fields on the exposed surface $(z=0)$, and temperature profiles at the boundaries $\left(x=0, x=l_{x}, y=0\right.$ and $\left.y=l_{y}\right)$, just after the excitation and at the exploitation limit, for the orthotropic material studied. 


\subsection{Estimation results}

\section{Isotropic material}

Due to the difficulty of assessing the performance of the method on a reference orthotropic material of perfectly known properties, the validation is conducted on an isotropic polymer sample whose thermal properties has been already identified by various method. The experimental procedure previously described, is conducted on a sample of thickness $l_{z}=2.4 \mathrm{~mm}$ measured by a digital micrometer, of heat capacity $C=$ $1670 \mathrm{~J} \cdot \mathrm{kg}^{-1} \cdot \mathrm{K}^{-1}$ measured by a Calvet calorimeter (C80 by Setaram(C) and a density of $\rho=$ $1140 \mathrm{~kg} \cdot \mathrm{m}^{-3}$ deduced from the weight and volume measurement of the sample. The predefined search space domain of the unknown diffusivities are restricted between $10^{-9}$ and $10^{-4} \mathrm{~m}^{2} . \mathrm{s}^{-1}$. The search space domain of the $R_{m, n}$ terms that describe the thermal excitation is $\left[-10^{3} ; 10^{3}\right]$. The even harmonics modes used for the estimation are $M=N=6$, so the corresponding modes are $m, n \in\{0,2,4,6\} \times\{0,2,4,6\}$, and the number of parameters to estimate is 19 . The dimension of the exploitation window are $l_{x}=28,0 \mathrm{~mm}$ and $l_{y}=26,1 \mathrm{~mm}$, centered on the laser impact.

Results obtained with various estimator are shown in Table 2. The diffusivity values obtained by the present estimation method (DSEH) shows a relatively low dispersion around the mean value (0.159), 2.5\%, 3.8\% and $-5.5 \%$ for the $\mathrm{x}, \mathrm{y}$ and $\mathrm{z}$ component, respectively. Considering the investigated material that has to be isotropic, the dispersion of the directional diffusivities seems to be consistent. The values are also coherent with the value founded by Santos [56] which gives for the Polyamide $a=0,147 \times 10^{-6} \mathrm{~m}^{2} \cdot \mathrm{s}^{-1}$. The results found by the present method along $\mathrm{x}$ and $\mathrm{y}$ axis are compared to those issued from a $2 \mathrm{D}$ reference estimation method known as ENH [22] [23], which has been numerically implemented for the purpose of the exercise. The relative difference between the 2 methods are small as it does not exceed $2 \%$. Finally, the estimated z-component value is compared to one obtained using the MSEH principle[25] based on the mean harmonic $(0,0)$ only. Once again, the agreement is quite convincing as the relative difference is below $1 \%$. These results confirm the perspective made by Ruffio in [28], where the potential of the DSEH method has been evoked from a theoretical point of view, without any implementation. 


\begin{tabular}{|c|c|c|c|c|c|}
\hline $\begin{array}{c}\text { Unknown } \\
\text { values }\end{array}$ & $\begin{array}{c}\text { DSEH } \\
\text { (present study) }\end{array}$ & $\begin{array}{c}\text { ENH } \\
{[22]}\end{array}$ & $\begin{array}{c}\text { Relative } \\
\text { difference }\end{array}$ & $\begin{array}{c}\text { MSEH } \\
{[25]}\end{array}$ & $\begin{array}{c}\text { Relative } \\
\text { difference }\end{array}$ \\
\hline$a_{x}$ & $0,163 \times 10^{-6}$ & $0,165 \times 10^{-6}$ & $1 \%$ & - & - \\
\hline$a_{y}$ & $0,165 \times 10^{-6}$ & $0,166 \times 10^{-6}$ & $<1 \%$ & - & - \\
\hline$a_{z}$ & $0,150 \times 10^{-6}$ & - & - & $0,147 \times 10^{-6}$ & $2 \%$ \\
\hline
\end{tabular}

Table 2-Comparison of the diffusivity values estimated by the Direct and Simultaneous Estimation using Harmonics (DSEH), with the values obtained by the Estimation using Normalization of Harmonics method (ENH) [22] and the Multiple Steps Estimation using Harmonics (MSEH) [25].

Erreur ! Source du renvoi introuvable. shows the normalized harmonics evolution as a function of time, resulting from both, the experiment, $\xi_{m, n}^{\exp }(t)$, and the simulation with the optimized parameters $\hat{b}$, $\xi_{m, n}(\hat{B}, t)$.

The comparison of the evolution shows a very close agreement between the signals. The relative error between the experimental and the estimated signals are calculated separately for each harmonics following matrix (8).

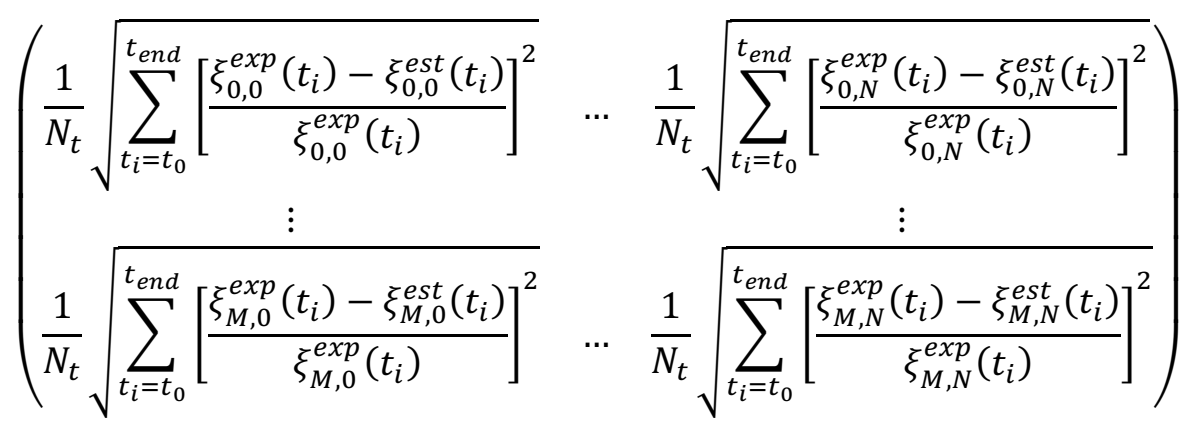

The calculated values $\sigma$ are indicated in Erreur ! Source du renvoi introuvable., the present material being referred as index 1 .

It is worth mentioning that $R_{0,0}=\mathrm{Q} \times F_{0,0}$, $\mathrm{Q}$ will be indirectly estimated due to the fact that $F_{0,0}$ is always equal to 1 (Fourier transform at frequency 0 ). Thus, the current method permits the estimation of not only the diffusivities, but also the total amount of energy Q, applied on the material.

For the polyamide sample, the total amount applied on the material is $Q=R_{0,0}=0.54 \mathrm{~J}$, in $\Delta t=10 \mathrm{~ms}$ corresponding to $41.5 \%$ of maximum laser capacity. 

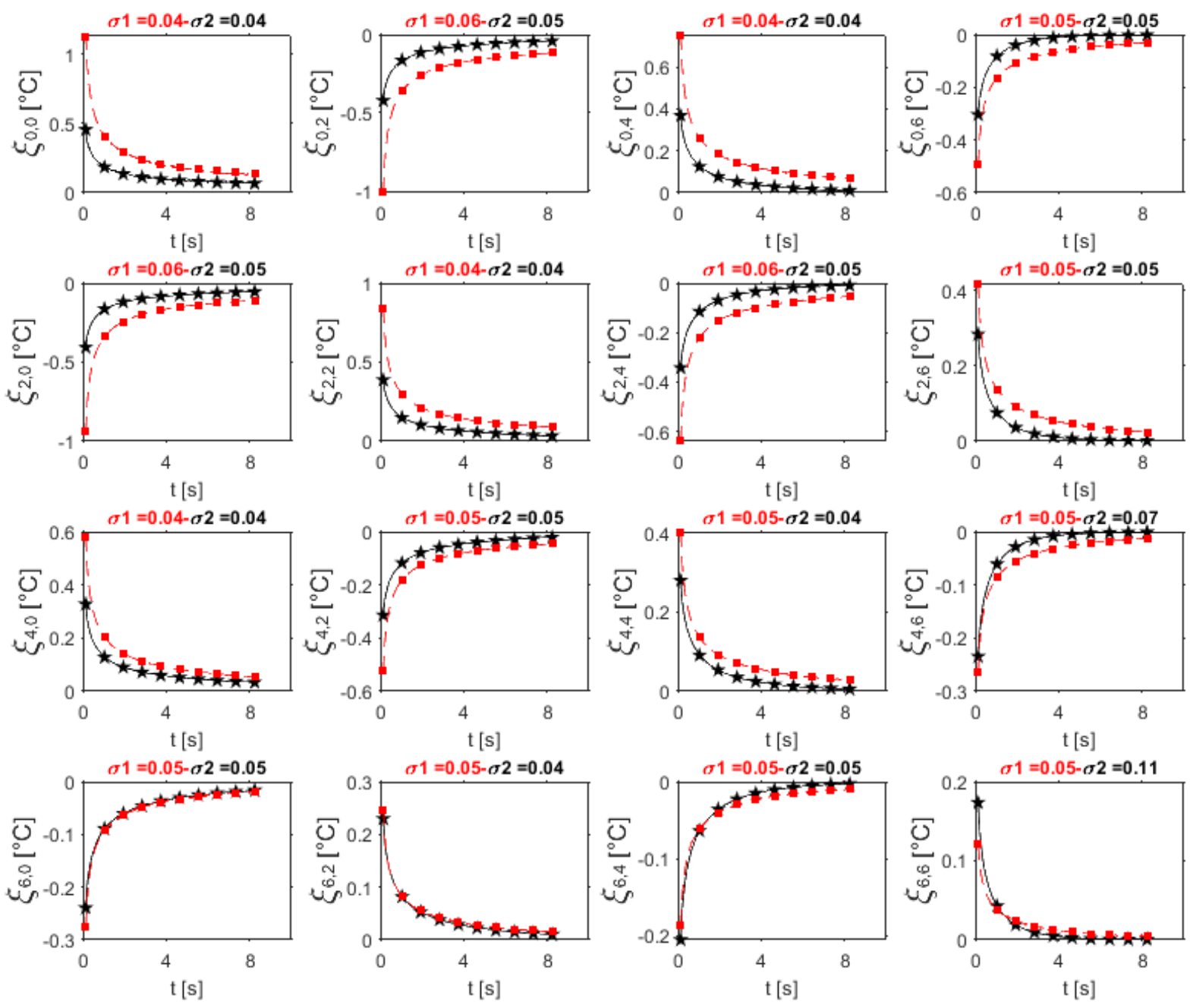

CMP-exp $\star$ CMP-est $---P A$-exp $\quad$ PA-est

Figure 6 : Normalized harmonics temporal evolution for experimental (dotted or normal lines) and inversely estimated data (symbols), applied for the polyamide -PA (red; square) - and the composite CMP (black; pentagram) -samples.

\section{Orthotropic material}

The orthotropic material studied in this section is a single embedded fiber composite, constituted of carbon fibers in an epoxy matrix. Orientation of the carbon fibers is the same along the material plane. The same experimental procedure as used in the validation section is conducted on a sample of thickness $l_{z}=$ $8.16 \mathrm{~mm}$, of heat capacity $C=1001 \mathrm{~J} \cdot \mathrm{kg}^{-1} \cdot \mathrm{K}^{-1}$ and a density of $\rho=1286 \mathrm{~kg} \cdot \mathrm{m}^{-3}$. The exploiting 
window is defined by $l_{x}=39.0 \mathrm{~mm}$ and $l_{y}=45.0 \mathrm{~mm}$, perfectly centered at the laser impact. Once again, Erreur! Source du renvoi introuvable. shows the excellent agreement between the experimental normalized harmonics, $\xi_{m, n}^{\text {exp }}(t)$ and the simulated harmonics obtained with the optimized parameters $\hat{b}$, $\xi_{m, n}(\hat{\theta}, t)$. The calculated values $\sigma$ are indicated in Erreur ! Source du renvoi introuvable., the present material being referred as index 2 . A comparison between values obtained by the present method with values obtained from various estimator are shown in Table 3. The relative deviation between the present estimator and the ENH estimator [22], reveals a relative difference about a $2.5 \%$ for $a_{x}$ and $1.5 \%$ for $a_{y}$. For $a_{z}$, the comparison is conducted with MSEH [25] and shows a relative difference of $4.5 \%$. Considering the carbon fibers being oriented along the y-axis, the ranking of the diffusivity components is consistent, $a_{y}>$ $a_{x}$ and $a_{z}$.

\begin{tabular}{|c|c|c|c|c|c|}
\hline $\begin{array}{c}\text { Unknown } \\
\text { values }\end{array}$ & $\begin{array}{c}\text { DSEH } \\
\text { (present study) }\end{array}$ & $\begin{array}{c}\text { ENH } \\
{[22]}\end{array}$ & $\begin{array}{c}\text { Relative } \\
\text { difference }\end{array}$ & $\begin{array}{c}\text { MSEH } \\
{[25]}\end{array}$ & $\begin{array}{c}\text { Relative } \\
\text { difference }\end{array}$ \\
\hline$a_{x}$ & $0.40 \times 10^{-6}$ & $0.39 \times 10^{-6}$ & $2.5 \%$ & - & \\
\hline$a_{y}$ & $2.59 \times 10^{-6}$ & $2.63 \times 10^{-6}$ & $1.5 \%$ & - & \\
\hline$a_{z}$ & $0.84 \times 10^{-6}$ & - & - & 0.88 & $4.5 \%$ \\
\hline $\mathrm{Q}$ & 0.71 & - & - & 0.73 & $2.7 \%$ \\
\hline
\end{tabular}

Table 3-Comparison of the diffusivity $\left(\mathrm{m}^{2} . \mathrm{s}^{-1}\right)$ and energy $Q(\mathrm{~J})$ values estimated by the Direct and Simultaneous Estimation using Harmonics (DSEH), with the values obtained by the Estimation using Normalization of Harmonics method (ENH) [22] and the Multiple Steps Estimation using Harmonics (MSEH) from [25].

The reproducibility of the algorithm is verified by running the code several times. The deviation of the obtained solutions does not exceed 5\%, whatever the parameter and despite the stochastic nature of the estimation method. Not shown here, the results obtained without taking into account the mean temperature field or the harmonic $(0,0)$, give the same results. This may be useful when we try to limit the surroundings influence. The robustness of the method is then maintained.

\section{Discussion and improvements}

In this section, the credibility of the previous estimation is conducted by means of a sensitivity study.

\subsection{Sensitivity analysis}

The sensitivity analysis is a key element, frequently used in the inverse problem to verify the feasibility of the estimation, to diagnose the corresponding best conditions required for identification, and to ensure the 
non-correlated nature of the parameters. It evaluates the influence of parameters to be estimated, or known a priori, on direct model outputs, here the harmonics $\xi_{\mathrm{m}, \mathrm{n}}(t, z=0)$. In the present case, this tool is used to insure the feasibility of the simultaneous estimation of the diffusivities. Thus, the variation of the front face normalized harmonics, $\partial \xi(6, t)$, caused by a relatively small variation in each parameter, $\partial B_{\mathbf{j}}$, is evaluated, while keeping all other parameters constant.

$$
\mathrm{S}_{m, n}\left(\beta_{\mathrm{j}}, \mathrm{t}\right)=\frac{\partial \xi_{\mathrm{m}, \mathrm{n}}(8, \mathrm{t})}{\partial \beta_{\mathrm{j}}} \times\left.\beta_{\mathrm{j}}\right|_{\beta_{\mathrm{k} \neq \mathrm{j}}}
$$

with, $B=\left[b_{1}, b_{2}, \ldots b_{n}\right]$ and $n$ is the number of parameters to be estimated. In this work, reduced sensitivities are considered, enabling the homogenization of the units (i.e. ${ }^{\circ} \mathrm{C}$ ) as well as the order of magnitude, and then facilitating the comparison between the parameters.

One should notice that the sensibility analysis requires the values of the parameters to estimate. A sensitivity study performed before the estimation step is then a qualitative evaluation rather than a quantitative one. A preliminary sensitivity study, conducted using supposed set of parameters in probable ranges of values, was used to set the optimization algorithm, particularly the time interval and the harmonics to exploit.

A sensitivity study is now performed using the values previously identified. Figure 7 and Figure 8 show the reduced sensitivity of the harmonics, during overall exploitation time, used for the estimation of the diffusivities. For example, the reduced sensitivity of harmonic $(0,0)$ to the diffusivity along the $\mathrm{x}$-axis is done using $\mathrm{S}_{0,0}\left(a_{x}, \mathrm{t}\right)=\frac{\partial \xi_{0,0}(8, \mathrm{t})}{\partial a_{x}} \times\left. a_{x}\right|_{\mathrm{B}_{\mathrm{k} \neq \mathrm{j}}}$. To evaluate sensitivities, time dependent harmonics gradients with respect to the unknown parameters, has to be treated. A large number of tests performed on various Laplace inversion techniques, shows that a coupled inversion technique was found to be the most appropriate for this exercise. It involves a Gaver-Stehfest method [57], more stable at short time, and a Hoog inversion [39] in the remaining time.

The reduced sensitivity of the harmonics, to the Polyamide diffusivities are plotted in Figure 7, and that for the composite are shown in Figure 8.

The reduced sensitivities analysis reveals the importance of each harmonics:

- $\xi_{0,0}$ brings information about $a_{z}$ only. This harmonic, corresponding to the mean temperature field, is also the most energetic.

- $\quad \xi_{m, 0}$ with $[0<m \leq 6, n=0]$, are only sensitive to $a_{x}$ and $a_{z}$ without any correlation.

- $\xi_{0, n}$ with $[m=0,0<n \leq 6]$, are only sensitive to $a_{y}$ and $a_{z}$ without any correlation. 
- $\xi_{m, n}$ with $[0<m \leq 6,0<n \leq 6]$ are sensitive to all diffusivities, but $a_{x}$ and $a_{y}$ exhibit a correlation, as revealed by the proportionality between those sensitivities. Thus, those harmonics does not carry any useful information for the estimation of these parameters. However, those harmonics are kept because it give additional information to estimate $a_{z}$ properly.
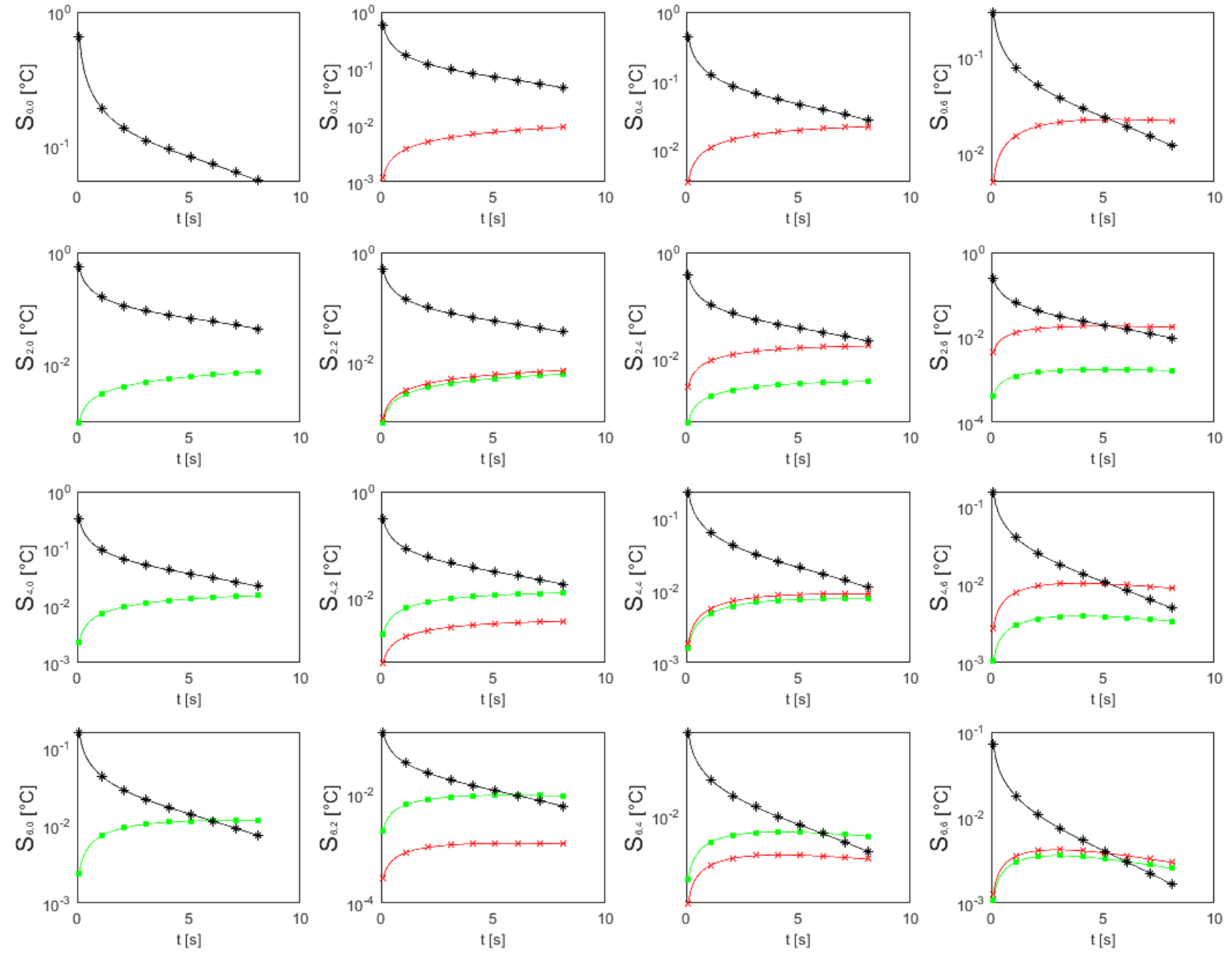

$\simeq \mathrm{S}_{\mathrm{m}, \mathrm{n}}(\mathrm{ax}, \mathrm{t}) \ldots \mathrm{S}_{\mathrm{m}, \mathrm{n}}(\mathrm{ay}, \mathrm{t}) \multimap \mathrm{S}_{\mathrm{m}, \mathrm{n}}(\mathrm{az}, \mathrm{t})$

Figure 7 : Sensitivities of the entire harmonics modes used for identification to the three main thermal diffusivities of the Polyamide sample. 

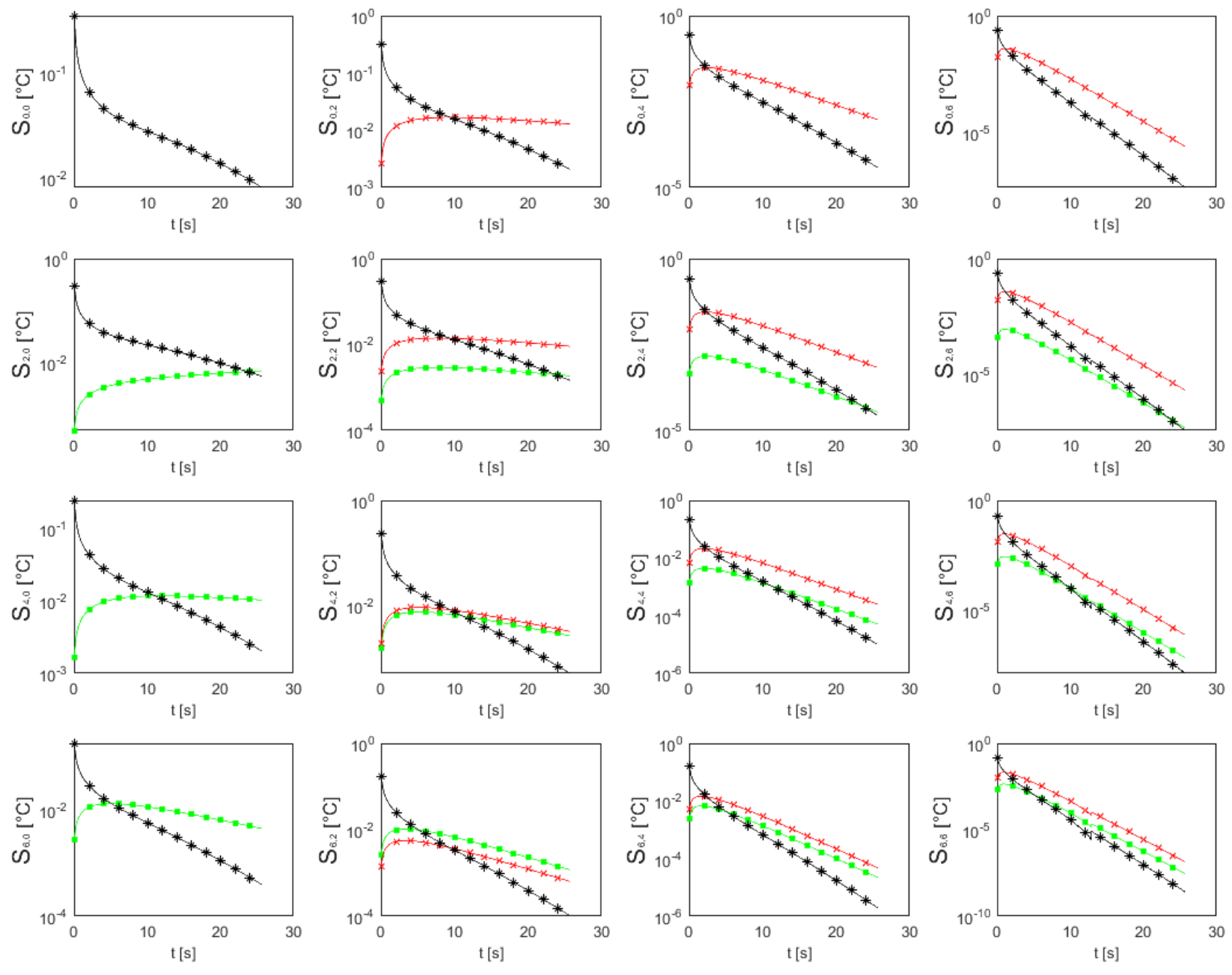

$=-S_{m, n}(a x, t) \multimap-S_{m, n}(a y, t) \multimap S_{m, n}(a z, t)$

Figure $8:$ Sensitivities of the entire harmonics modes used for identification to the three main thermal diffusivities of the composite sample.

\section{Sensitivity to the overall heat transfer coefficient}

The heat exchange, involving radiation and convection modes, between the environments and the materials are considered to take place at the exposed and rear faces of the samples, only. The value of the overall coefficient, obtained by linearization of the radiative term, is assumed to be equal on both sides and represented by the constant coefficient $h$. As a first guess, this value is taken to be $h=10 \mathrm{~W} \cdot \mathrm{m}^{-2} \cdot \mathrm{K}^{-1}$, which is commonly admit for such environmental conditions.

To highlight the negligible influence of overall heat transfer coefficient on the front face temperature, the front face signal issued form the analytical model, has been plotted for different extended values of $h \in$ $\{0,5,10,15,20\}$ in Figure 9 using parameters consistent with experimental conditions. 
As shown in Figure 9, the value of the overall coefficient has no effect on the first 4 harmonics during the considered time of exploitation $(t<25 s)$.

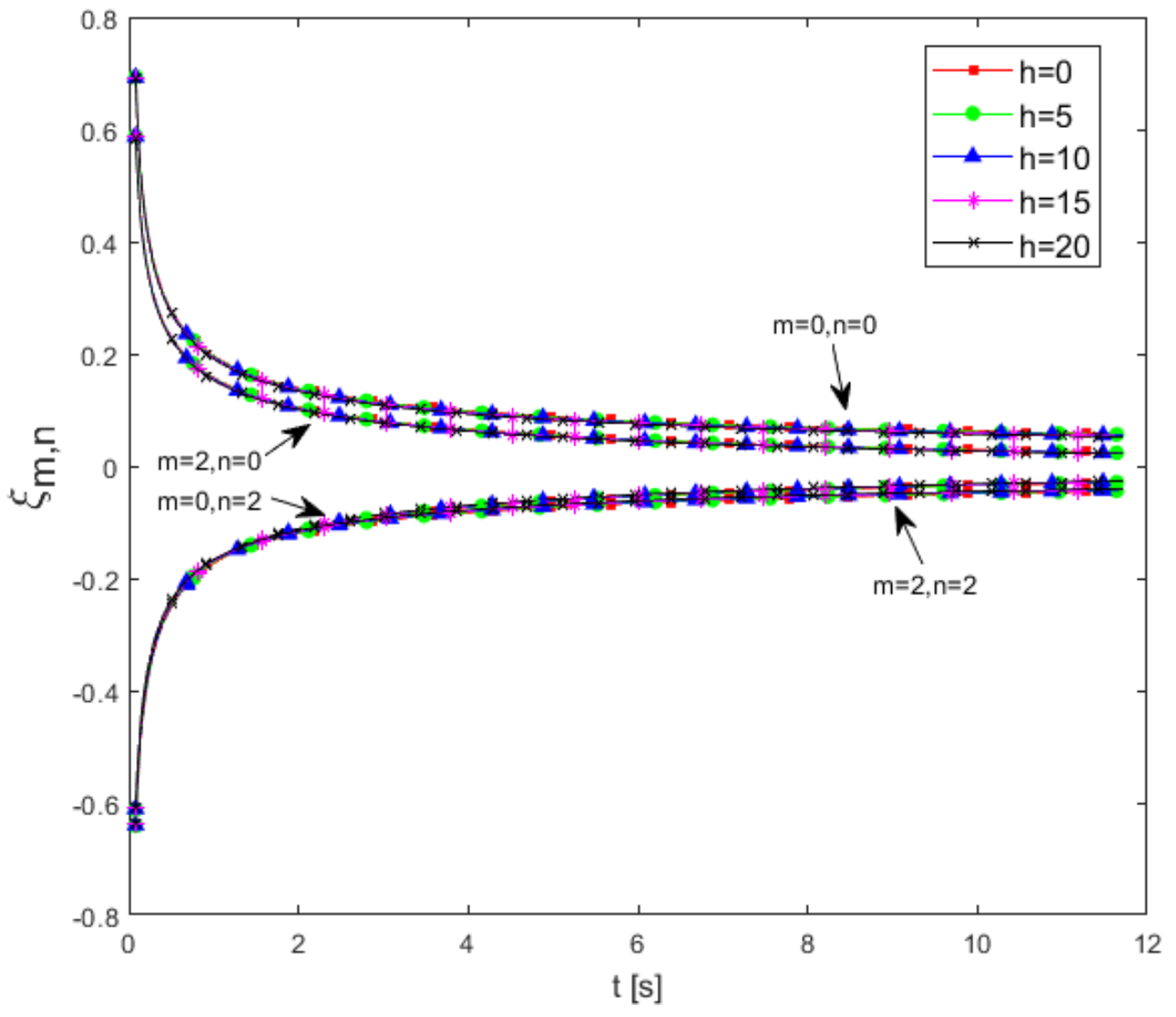

Figure 9 : Evolution of the first 4 harmonics, according to the value of the overall heat coefficient $h$.

\subsection{Improvements}

As shown previously, the time consumed through this estimation, which relies on an analytical model, is quite moderate, compared to numerical methods. Nevertheless, an improvement may be achieved, while conserving a certain level of accuracy. Regarding that time consumption sources, the PSO minimization section has proved to be the more consuming. The main objective of this section is to reduce that time, by envisaging many possible strategies.

- Filtering the images required for the identification, this strategy does not reduce significantly the computational time.

- Reducing the number of PSO particles will decrease the CPU time but makes it vulnerable to local convergence and some authors prescribed the number of particle to be set as 10 times the number of parameter to identify. 
- Decreasing the number of exploitation harmonics. For example, changing from a number of harmonics $\mathrm{M}=\mathrm{N}=6$ to $\mathrm{M}=\mathrm{N}=4$, reduces the number of parameter to estimation from 19 to 12 . This still conduct to precise results but does not reduce the calculation time significantly.

- Imposing the shape of the excitation that, in turn, may significantly reduce the number of parameters to identify. Currently, the identification of the 3 thermal diffusivities requires the estimation of 16 parameters related to the form of the excitation. Thus, the reduction of the number of parameters needed to mimic the excitation is investigated in this part. One strategy consists in considering the excitation with an a priori knowledge of its shape. In this case, the number of parameters is reduced to the number of parameters of the analytical function describing the prescribed flux distribution. Although difficult to describe, several laser excitation forms can be found in literature [28]: Gaussian, parabolic, triangular, uniform, pointed, etc.

In this work, according to the first IR camera images, two excitation shapes seem to be physically coherent and are tested. The shape function associated with the laser beam, $F_{x, y}$, is assumed to follow a cosine function or a polynomial cubic form. Figure 10 represents the temperature evolution when having these two possible shapes of laser beam.

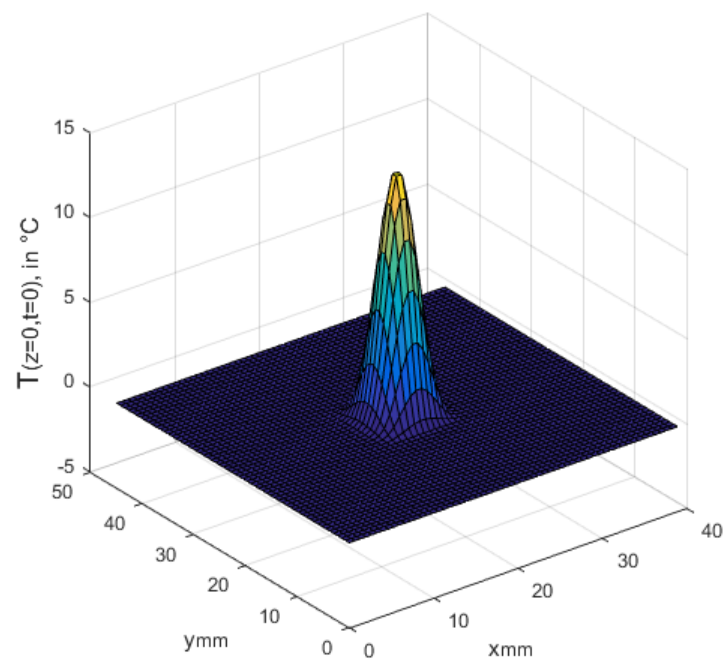

a)

Figure 10 : Temperature elevation fields at $t=t_{0}$ for the a) cosine and b) cubic polynomial predefined shapes.

\section{Cosine function}

The shape of the excitation is described, along the $\mathrm{x}$-axis, by the function 


$$
f(x)=\frac{1}{r} \times\left\{\begin{array}{cl}
\frac{1}{2}\left(1+\cos \left(\frac{\pi x}{r}\right)\right) & \text { for }-r<x<r \\
0 & \text { otherwise }
\end{array}\right.
$$

with $r$ the radius of the laser spot. The Fourier transform of the previous function is

$$
f_{m}=\int_{0}^{l x} f\left(x-\frac{l x}{2}\right) \cos \left(\frac{m \pi x}{l_{x}}\right) d x=\frac{1}{r} \cdot \frac{l_{x}{ }^{3} \cdot \cos \left(\frac{m \cdot \pi}{2}\right) \cdot \sin \left(\alpha_{m} \cdot r\right)}{l_{x}{ }^{2} \cdot m \cdot \pi-m^{3} \cdot \pi \cdot r^{2}}
$$

The same function is used for $\mathrm{f}(\mathrm{y})$. And following that, the shape function associated with the laser beam, $F_{x, y}=f(x) \times f(y)$, in the Fourier domain, leads to the dimensionless shape factor:

$$
F_{m, n}=\frac{1}{r^{2} \cdot \pi}\left(\frac{l_{x}{ }^{3} \cdot l_{y}{ }^{3} \cdot \cos \left(\frac{m \pi}{2}\right) \cdot \cos \left(\frac{n \pi}{2}\right) \cdot \sin \left(\alpha_{m} r\right) \cdot \sin \left(\beta_{n} r\right)}{\left(l_{x}{ }^{2} m-m^{3} r^{2}\right) \cdot\left(l_{y}{ }^{2} n-n^{3} r^{2}\right)}\right)
$$

\section{Polynomial cubic}

The shape of the excitation is described, along the $\mathrm{x}$-axis,

$$
f(x)=\frac{1}{r}\left\{\begin{array}{cl}
1-3\left(\frac{x}{r}\right)^{2}-2\left(\frac{x}{r}\right)^{3} & \text { for } \quad-r \leq x<0 \\
1-3\left(\frac{x}{r}\right)^{2}+2\left(\frac{x}{r}\right)^{3} & \text { for } 0 \leq x<r \\
0 & \text { otherwise }
\end{array}\right.
$$

The Fourier transform of the previous function is

$$
\begin{aligned}
f_{m}=\int_{0}^{l x} f\left(x-\frac{l x}{2}\right) \cos \left(\frac{m \pi x}{l_{x}}\right) d x \\
=\frac{-24\left(\cos \left(\frac{m \pi}{2}\right) \times\left(\cos \left(\alpha_{m} r\right)-1+\frac{\alpha_{m} r}{2} \times \sin \left(\alpha_{m} r\right)\right)\right.}{r^{4} \alpha_{m}{ }^{4}}
\end{aligned}
$$

The same function is used for $\mathrm{f}(\mathrm{y})$. Consequently, the shape function associated with the laser beam, $F_{x, y}=$ $f(x) \times f(y)$, in the Fourier domain, leads to the dimensionless shape factor:

$$
\begin{gathered}
F_{m, n}=\frac{24^{2}}{r^{8} \cdot \alpha_{m}{ }^{4} \cdot \beta_{n}{ }^{4}}\left[\left(\cos \left(\frac{m \pi}{2}\right) \times\left(\cos \left(\alpha_{m} r\right)-1+\frac{\alpha_{m} r}{2} \times \sin \left(\alpha_{m} r\right)\right)\right.\right. \\
\times\left(\cos \left(\frac{n \pi}{2}\right) \times\left(\cos \left(\beta_{n} r\right)-1+\frac{\beta_{n} r}{2} \times \sin \left(\beta_{n} r\right)\right)\right]
\end{gathered}
$$


Considering any of the 2 predefined shapes, defined in equation (15) and (18), the only unknown parameter is the laser radius $r$. The excitation distribution, described by $\emptyset_{m n, p}^{e x}=Q \cdot F_{m, n} \cdot u(p)$, that appears in equation (8) ,implies the knowledge of the excitation intensity $Q$, which is also identified in the present version of the method. Therefore, the parameters vector is $B=\left[a_{x}, a_{y}, a_{z}, Q, r\right]$.

For comparative purposes, the estimation part was applied on the orthotropic material and performed using the same PSO parameters. As shown in Table 4, the results obtained are quite similar whatever the presupposed shape, as well as the calculation time is one order of magnitude lower than the original estimation. Concerning the estimated values, the level of accuracy is acceptable as the relative error of the global estimation (i.e. the summation of the independent relative error) does not exceed $8 \%$.

The present strategy, could be conducted as a first guess approach, whose results may be used to initialize and ranging the unknown parameters of the original method in a closer search domain.

\begin{tabular}{|c|c|c|c|c|c|c|}
\hline $\begin{array}{c}\text { Unknown } \\
\text { values }\end{array}$ & $\begin{array}{c}\text { Non-predefined } \\
\text { shape (6 harm) }\end{array}$ & $\begin{array}{c}\text { Non-predefined } \\
\text { shape (4 harm) }\end{array}$ & $\begin{array}{c}\text { Cubic form } \\
(6 \text { harm })\end{array}$ & $\begin{array}{c}\text { Cosine form } \\
(6 \text { harm })\end{array}$ & $\begin{array}{c}\text { Cosine form } \\
(4 \text { harm })\end{array}$ & $\begin{array}{c}\text { Cubic form } \\
(4 \text { harm })\end{array}$ \\
\hline$a_{x} \times 10^{-6}$ & 0.40 & 0.411 & 0.504 & 0.500 & 0.485 & 0.485 \\
\hline$a_{y} \times 10^{-6}$ & 2.59 & 2.610 & 2.370 & 2.370 & 2.47 & 2.47 \\
\hline$a_{z} \times 10^{-6}$ & 0.84 & 0.828 & 0.901 & 0.902 & 0.856 & 0.857 \\
\hline $\mathrm{Q}$ & 0.717 & 0.714 & 0.714 & 0.726 & 0.712 & 0.708 \\
\hline$t_{C P U}$ & $35 \mathrm{~min}$ & $25 \mathrm{~min}$ & $3 \mathrm{~min}$ & $3 \mathrm{~min}$ & 2 min & 2 min \\
\hline
\end{tabular}

Table 4- Summary of the diffusivities $\left(\mathrm{m}^{2} \cdot \mathrm{s}^{-1}\right)$ and energy $Q(J)$ estimation as a function of the excitation form.

Besides the estimation of the diffusivities, this version of methods can also identify the value of the amount of energy absorbed by the sample $Q$, and the spot radius $r$.

In both cases (cubic or cosine shapes), the values identified are the same, with $Q=0.71 \mathrm{~J}$ and $r=5.6 \mathrm{~mm}$. The value of $Q$ corresponds to $55 \%$ of the maximum laser capacity (130W) which is coherent with the laser settings. The value of $r$ corresponds roughly to the observation on the first IR images, illustrated in Figure 4. Those results, $B=\left[a_{x}, a_{y}, a_{z}, Q, r\right]$, may be used as is, when the constraints may require a fast estimation (i.e. quality control in manufacturing), or as a preliminary guess in order to restrict the search domains. 
For this purpose a two-steps method was conducted. It firstly estimate a vector $b^{\prime}=\left[a_{x}{ }^{\prime}, a_{y}{ }^{\prime}, a_{z}{ }^{\prime}, Q, r\right]$ by applying a predefined shape (cosine or cubic).Then the search space domain of the original method (with a non-predefined shape), is re-initialized with a more-restricted area, using the resulted vector $b^{\prime}$,obtained using the short preliminary step with predefined excitation shape.

Having $F_{m, n}$ which is always between -1 and $1, R_{m, n}$ will be between $-\mathrm{Q}$ and Q. The new search space domain for $\left[a_{x}, a_{y}, a_{z}, R_{m, n}\right]$ will have a lower bound of $\left[a_{x}{ }^{\prime} / 2, a_{y}{ }^{\prime} / 2, a_{z}{ }^{\prime} / 2,-2 Q\right]$ and an upper bound of $\left[2 a_{x}{ }^{\prime}, 2 a_{y}{ }^{\prime}, 2 a_{z}{ }^{\prime}, 2 Q\right]$; The results of this two-steps method are represented in Table 5 and compared to the results of one-step method with non-predefined shape of the laser beam.

\begin{tabular}{|c|c|c|}
\hline Unknown values & $\begin{array}{c}\text { Non-predefined shape } \\
(6 \text { harm })\end{array}$ & $\begin{array}{c}\text { Two-steps method } \\
(6 \text { harm })\end{array}$ \\
\hline$a_{x} \times 10^{-6}$ & 0.40 & 0.40 \\
\hline$a_{y} \times 10^{-6}$ & 2.59 & 2.59 \\
\hline$a_{z} \times 10^{-6}$ & 0.84 & 0.84 \\
\hline $\mathrm{Q}$ & 0.717 & 0.718 \\
\hline$t_{C P U}$ & $35 \mathrm{~min}$ & $18 \mathrm{~min}$ \\
\hline
\end{tabular}

Table 5 : Comparison of the results between the one-step (non-predefined) and two-step methods

\section{Conclusion}

In this work, an identification method devoted to the thermal characterization of an isotropic or an orthotropic material is presented. The originality of this method lies in the possibility to directly and simultaneously estimate, in only one calculation step, the three main diffusivities of any orthotropic or isotropic material. This identification procedure relies on an analytical resolution of the heat transfer equation, on the non-intrusive measurements of the temperature field induced by a local excitation. Only one experiment is required to achieve this goal.

It is well known that the parameters relative to the excitation are highly dependent on the laser beam shape which is usually difficult to identify. In this context, one of the distinctive features of our approach, is that the estimation may be successfully achieved without any a priori knowledge about the shape or the intensity of the excitation. Adding to that, it estimates, simultaneously with the thermal diffusivities, the total amount of heat absorbed by the material, and predicts the shape of the thermal excitation applied on the surface of the material. 
In addition, the method being based on an analytical model, it allows the use of a stochastic approach that would be faster in this case. And the results obtained demonstrate that the hybrid optimization strategy combining a PSO algorithm and a gradient based method is a good candidate for resolving such complex and non-linear inverse problem. After validation with isotropic materials of known properties, this method is applied on an orthotropic fiber composite material. The very good agreement between the results in the present study and results obtained from other estimators (e.g. ENH, MSEH) is shown and demonstrate the accuracy of the method. Finally, the reproducibility and the robustness of the method is demonstrated.

Based on these results, this method appears to be very promising in order to identify thermophysical properties of multilayer orthotropic materials that will be addressed in a future work.

\section{Acknowledgements}

The Authors thank the European Union and the Nouvelle Aquitaine District for their financial support through the CPER/FEDER 2014-2020 program.

\section{References}

[1] A. Degiovanni, Conductivite et diffusivite thermique des solides. Ed. Techniques Ingénieur.

[2] C. Rodiet, "Temperature Measurement by Multi-Spectral Methods and Thermal Characterization of Anisotropic Materials by Integral Transforms: Theoretical and experimental aspects," Theses, Université de Lorraine; Institut National Polytechnique de Lorraine (INPL), 2014.

[3] W. J. Parker, R. J. Jenkins, C. P. Butler, and G. L. Abbott, "Flash Method of Determining Thermal Diffusivity, Heat Capacity, and Thermal Conductivity," J. Appl. Phys., vol. 32, no. 9, pp. 1679-1684, Sep. 1961.

[4] A. B. Donaldson and R. E. Taylor, "Thermal diffusivity measurement by a radial heat flow method," J. Appl. Phys., vol. 46, no. 10, pp. 4584-4589, Oct. 1975.

[5] M. Amazouz, Moyne, and A. Degiovanni, "Measurement of the thermal diffusivity of anisotropic materials," High Temperatures. High Pressures, pp. 37-41, 1987.

[6] A. Degiovanni, J. C. Batsale, and D. Maillet, "Mesure de la diffusivité longitudinale de matériaux anisotropes," Rev. Générale Therm., vol. 35, no. 410, pp. 141-147, Feb. 1996.

[7] S. Graham, D. L. McDowell, and R. B. Dinwiddie, "Multidimensional Flash Diffusivity Measurements of Orthotropic Materials," Int. J. Thermophys., vol. 20, no. 2, pp. 691-707, Mar. 1999.

[8] J. Beňačka, L. Vozár, and I. Štubňa, "Design of Experiment for Thermal Diffusivity Measurements of Composite Material with Orthogonal Anisotropy," Int. J. Thermophys., vol. 29, no. 6, pp. 2088-2101, Dec. 2008.

[9] D. Demange, P. Beauchêne, M. Bejet, and R. Casulleras, "Mesure simultanée de la diffusivité thermique selon les deux directions principales d'un matériau," Rev. Générale Therm., vol. 36, no. 10, pp. 755-770, Nov. 1997. 
[10] B. Sawaf and M. N. Özisik, "Determining the constant thermal conductivities of orthotropic materials by inverse analysis," Int. Commun. Heat Mass Transf., vol. 22, no. 2, pp. 201-211, Mar. 1995.

[11] S. Chanda, C. Balaji, S. P. Venkateshan, A. Ambirajan, and V. Ramakrishnan, "Simultaneous Estimation of Principal Thermal Conductivities of an Anisotropic Composite Medium: An Inverse Analysis," J. Heat Transf., vol. 135, no. 2, pp. 021301-021301-8, Jan. 2013.

[12] V. Plana, P. Reulet, and P. Millan, "Experimental Characterization of the Thermophysical Properties of Composite Materials by an Inverse Heat Conduction Method," J. Compos. Mater., vol. 40, no. 14, pp. 1247-1258, Jul. 2006.

[13] M. Thomas, N. Boyard, N. Lefèvre, Y. Jarny, and D. Delaunay, "An experimental device for the simultaneous estimation of the thermal conductivity 3-D tensor and the specific heat of orthotropic composite materials," Int. J. Heat Mass Transf., vol. 53, no. 23, pp. 5487-5498, Nov. 2010.

[14] W. P. Adamczyk, R. A. Białecki, and T. Kruczek, "Retrieving thermal conductivities of isotropic and orthotropic materials," Appl. Math. Model., vol. 40, no. 4, pp. 3410-3421, Feb. 2016.

[15] W. P.Adamczyk, R. A.Białecki, and T. Kruczek, "Measuring thermal conductivity tensor of orthotropic solid bodies - ScienceDirect," Measurement, pp. 93-102, Apr-2017.

[16] N. S. Mera, L. Elliott, D. B. Ingham, and D. Lesnic, "Use of the boundary element method to determine the thermal conductivity tensor of an anisotropic medium," Int. J. Heat Mass Transf., vol. 44, no. 21, pp. 4157-4167, Nov. 2001.

[17] M. R. Hematiyan, A. Khosravifard, and Y. C. Shiah, "A novel inverse method for identification of 3D thermal conductivity coefficients of anisotropic media by the boundary element analysis," Int. J. Heat Mass Transf., vol. 89, no. Supplement C, pp. 685-693, Oct. 2015.

[18] B. Chen, W. Chen, A. H.-D. Cheng, L.-L. Sun, X. Wei, and H. Peng, "Identification of the thermal conductivity coefficients of 3D anisotropic media by the singular boundary method," Int. J. Heat Mass Transf., vol. 100, pp. 24-33, Sep. 2016.

[19] I. Philippi, J. C. Batsale, D. Maillet, and A. Degiovanni, "Measurement of thermal diffusivities through processing of infrared images," Rev. Sci. Instrum., vol. 66, no. 1, pp. 182-192, Jan. 1995.

[20] B. Remy, A. Degiovanni, and D. Maillet, "Mesure de la diffusivité thermique de matériaux anisotropes de petites dimensions par thermographie infrarouge et transformations intégrales," in SFT, 2007.

[21] B. Rémy, A. Degiovanni, and D. Maillet, "Measurement of the In-plane Thermal Diffusivity of Materials by Infrared Thermography," Int. J. Thermophys., vol. 26, no. 2, pp. 493-505, Mar. 2005.

[22] J.-C. Krapez, L. Spagnolo, M. Frieß, H.-P. Maier, and G. Neuer, "Measurement of in-plane diffusivity in non-homogeneous slabs by applying flash thermography," Int. J. Therm. Sci., vol. 43, no. 10, pp. 967-977, Oct. 2004.

[23] P. Bison, F. Cernuschi, and E. Grinzato, "In-depth and In-plane Thermal Diffusivity Measurements of Thermal Barrier Coatings by IR Camera: Evaluation of Ageing," Int. J. Thermophys., vol. 29, no. 6, pp. 2149-2161, Dec. 2008.

[24] V. Vavilov, D. Burleigh, and V. Shiryaev, "IR thermographic evaluation of thermal diffusivity anisotropy: comparative analysis of some algorithms," Quant. InfraRed Thermogr. J., vol. 4, no. 2, pp. 187-200, Dec. 2007.

[25] Y. Souhar, B. Remy, and A. Degiovanni, "High Temperature Facility Under Vacuum for the Thermal Characterization of Anisotropic Materials," Proc. Int Heat Transf. Conf. - IHTC14, pp. 381-386, Jan. 2010.

[26] C. Rodiet, B. Remy, and A. Degiovanni, “Thermal characterization of anisotropic materials by integral transforms taking into account the thermal coupling with the sample-holder," Int. J. Therm. Sci., vol. 79, no. Supplement C, pp. 67-75, May 2014.

[27] T. Tian and K. D. Cole, "Anisotropic thermal conductivity measurement of carbon-fiber/epoxy composite materials," Int. J. Heat Mass Transf., vol. 55, no. 23, pp. 6530-6537, Nov. 2012.

[28] E. Ruffio, D. Saury, and D. Petit, "Improvement and comparison of some estimators dedicated to thermal diffusivity estimation of orthotropic materials with the 3D-flash method," Int. J. Heat Mass Transf., vol. 64, no. Supplement C, pp. 1064-1081, Sep. 2013. 
[29] H. Qi, C.-Y. Niu, S. Gong, Y.-T. Ren, and L.-M. Ruan, "Application of the hybrid particle swarm optimization algorithms for simultaneous estimation of multi-parameters in a transient conductionradiation problem," Int. J. Heat Mass Transf., vol. 83, pp. 428-440, Apr. 2015.

[30] E. Ruffio, D. Saury, and D. Petit, "Robust experiment design for the estimation of thermophysical parameters using stochastic algorithms," Int. J. Heat Mass Transf., vol. 55, no. 11, pp. 2901-2915, May 2012.

[31] R. V. Rao and V. K. Patel, "Thermodynamic optimization of cross flow plate-fin heat exchanger using a particle swarm optimization algorithm," Int. J. Therm. Sci., vol. 49, no. 9, pp. 1712-1721, Sep. 2010.

[32] R. Das, K. Singh, B. Akay, and T. Gogoi, "Application of artificial bee colony algorithm for maximizing heat transfer in a perforated fin," Proc. Inst. Mech. Eng. Part E J. Process Mech. Eng., vol. 232, no. 1, pp. 38-48, Feb. 2018.

[33] B. Zhang, H. Qi, Y.-T. Ren, S.-C. Sun, and L.-M. Ruan, "Application of homogenous continuous Ant Colony Optimization algorithm to inverse problem of one-dimensional coupled radiation and conduction heat transfer," Int. J. Heat Mass Transf., vol. 66, pp. 507-516, Nov. 2013.

[34] R. Das, K. Singh, and T. K. Gogoi, "Estimation of critical dimensions for a trapezoidal-shaped steel fin using hybrid differential evolution algorithm," Neural Comput. Appl., vol. 28, no. 7, pp. 16831693, Jul. 2017.

[35] S. Chanda, C. Balaji, S. P. Venkateshan, and G. R. Yenni, "Estimation of principal thermal conductivities of layered honeycomb composites using ANN-GA based inverse technique," Int. J. Therm. Sci., vol. 111, no. Supplement C, pp. 423-436, Jan. 2017.

[36] S. Orain, Y. Scudeller, S. Garcia, and T. Brousse, "Use of genetic algorithms for the simultaneous estimation of thin films thermal conductivity and contact resistances," Int. J. Heat Mass Transf., vol. 44, no. 20, pp. 3973-3984, Oct. 2001.

[37] Y. Billaud et al., "A hybrid small-world network/semi-physical model for predicting wildfire spread in heterogeneous landscapes," J. Phys. Conf. Ser., vol. 395, no. 1, p. 012008, 2012.

[38] D. Maillet, S. André, J. C. Batsale, A. Degiovanni, and C. Moyne, Thermal quadrupoles: solving the heat equation through integral transforms. Wiley, 2000.

[39] F. de Hoog, J. Knight, and A. Stokes, "An Improved Method for Numerical Inversion of Laplace Transforms," SIAM J. Sci. Stat. Comput., vol. 3, no. 3, pp. 357-366, Sep. 1982.

[40] A. Khare and S. Rangnekar, "A review of particle swarm optimization and its applications in Solar Photovoltaic system," Appl. Soft Comput., vol. 13, no. 5, pp. 2997-3006, May 2013.

[41] Z.-L. Gaing, "A Particle Swarm Optimization Approach for Optimum Design of PID Controller in AVR System,” IEEE Trans. Energy Convers., vol. 19, no. 2, pp. 384-391, Jun. 2004.

[42] M. Y. Hassan, M. N. Suharto, M. P. Abdullah, M. S. Majid, and F. Hussin, "Application of Particle Swarm Optimization for Solving Optimal Generation Plant Location Problem,” p. 10, 2012.

[43] B. Mohammadi-Ivatloo, M. Moradi-Dalvand, and A. Rabiee, "Combined heat and power economic dispatch problem solution using particle swarm optimization with time varying acceleration coefficients," Electr. Power Syst. Res., vol. 95, pp. 9-18, Feb. 2013.

[44] M. R. AlRashidi and M. E. El-Hawary, "A Survey of Particle Swarm Optimization Applications in Electric Power Systems," IEEE Trans. Evol. Comput., vol. 13, no. 4, pp. 913-918, Aug. 2009.

[45] N. Jin and Y. Rahmat-Samii, "Particle Swarm Optimization for Antenna Designs in Engineering Electromagnetics," J Artif Evol App, vol. 2008, pp. 9:1-9:10, Jan. 2008.

[46] Eberhart and Y. Shi, "Particle swarm optimization: developments, applications and resources," in Proceedings of the 2001 Congress on Evolutionary Computation (IEEE Cat. No.01TH8546), 2001, vol. 1, pp. 81-86 vol. 1.

[47] Y. del Valle, G. K. Venayagamoorthy, S. Mohagheghi, J. Hernandez, and R. G. Harley, "Particle Swarm Optimization: Basic Concepts, Variants and Applications in Power Systems," IEEE Trans. Evol. Comput., vol. 12, no. 2, pp. 171-195, Apr. 2008. 
[48] B. Zhang, H. Qi, S.-C. Sun, L.-M. Ruan, and H.-P. Tan, "Solving inverse problems of radiative heat transfer and phase change in semitransparent medium by using Improved Quantum Particle Swarm Optimization,” Int. J. Heat Mass Transf., vol. 85, pp. 300-310, Jun. 2015.

[49] S. Soleimani, D. D. Ganji, M. Gorji, H. Bararnia, and E. Ghasemi, "Optimal location of a pair heat source-sink in an enclosed square cavity with natural convection through PSO algorithm," Int. Commun. Heat Mass Transf., vol. 38, no. 5, pp. 652-658, May 2011.

[50] V. K. Patel and R. V. Rao, "Design optimization of shell-and-tube heat exchanger using particle swarm optimization technique," Appl. Therm. Eng., vol. 30, no. 11, pp. 1417-1425, Aug. 2010.

[51] K. H. Lee, S. W. Baek, and K. W. Kim, "Inverse radiation analysis using repulsive particle swarm optimization algorithm," Int. J. Heat Mass Transf., vol. 51, no. 11, pp. 2772-2783, Jun. 2008.

[52] J. Kennedy, "Swarm Intelligence," in Handbook of Nature-Inspired and Innovative Computing, Springer, Boston, MA, 2006, pp. 187-219.

[53] Q. Bai, "Analysis of Particle Swarm Optimization Algorithm," Comput. Inf. Sci., vol. 3, no. 1, p. 180, Jan. 2010.

[54] I. C. Trelea, "The particle swarm optimization algorithm: convergence analysis and parameter selection," Inf. Process. Lett., vol. 85, no. 6, pp. 317-325, Mar. 2003.

[55] R.A. Waltz, J.L. Morales, J. Nocedal, and D. Orban, "An interior algorithm for nonlinear optimization that combines line search and trust region steps." .

[56] W.Santos, P.Mummery, and A.Wallwork, "Thermal diffusivity of polymers by the laser flash technique - ScienceDirect," Polymer Testing, Aug-2005.

[57] H. Stehfest, "Algorithm 368: Numerical Inversion of Laplace Transforms [D5]," Commun ACM, vol. 13, no. 1, pp. 47-49, Jan. 1970. 